\title{
Article \\ Solving a System of Nonlinear Integral Equations via Common Fixed Point Theorems on Bicomplex Partial Metric Space
}

\author{
Zhaohui Gu ${ }^{1, *}$, Gunaseelan Mani ${ }^{2}\left(\mathbb{D}\right.$, Arul Joseph Gnanaprakasam ${ }^{3}\left(\mathbb{C}\right.$ and Yongjin $\mathrm{Li}^{4}(\mathbb{C}$ \\ 1 School of Mathematics and Statistics, Guangdong University of Foreign Studies, Guangzhou 510006, China \\ 2 Department of Mathematics, Sri Sankara Arts and Science College (Autonomous), Affiliated to \\ Madras University, Enathur, Kanchipuram 631 561, Tamil Nadu, India; mathsguna@yahoo.com \\ 3 Department of Mathematics, Faculty of Engineering and Technology, College of Engineering and Technology, \\ SRM Institute of Science and Technology, SRM Nagar, Kattankulathur 603 203, Tamil Nadu, India; \\ aruljoseph.alex@gmail.com \\ 4 Department of Mathematics, Sun Yat-Sen University, Guangzhou 510275, China; stslyj@mail.sysu.edu.cn \\ * Correspondence: zhgugz@163.com
}

check for updates

Citation: Gu, Z.; Mani, G.;

Gnanaprakasam, A.J.; Li, Y. Solving a

System of Nonlinear Integral

Equations via Common Fixed Point

Theorems on Bicomplex Partial Metric Space. Mathematics 2021, 9 , 1584. https://doi.org/10.3390/ math9141584

Academic Editor: Christopher Goodrich

Received: 18 June 2021

Accepted: 28 June 2021

Published: 6 July 2021

Publisher's Note: MDPI stays neutral with regard to jurisdictional claims in published maps and institutional affiliations.

Copyright: (c) 2021 by the authors. Licensee MDPI, Basel, Switzerland. This article is an open access article distributed under the terms and conditions of the Creative Commons Attribution (CC BY) license (https:/ / creativecommons.org/licenses/by/ $4.0 /)$.

\begin{abstract}
In this paper, we introduce the notion of bicomplex partial metric space and prove some common fixed point theorems. The presented results generalize and expand some of the literature's well-known results. An example and application on bicomplex partial metric space is given.
\end{abstract}

Keywords: integral equations; bicomplex partial metric space; common fixed point

MSC: 47H9; 47H10; 30G35; 46N99; 54H25

\section{Introduction}

Serge [1] made a pioneering attempt in the development of special algebra. $\mathrm{He}$ conceptualized commutative generalization of complex numbers, bicomplex numbers, tricomplex numbers, etc. as elements of an infinite set of algebra. Subsequently, in the 1930s, researchers contributed in this area [2-4]. The next fifty years failed to witness any advancement in this field. Later, Price [5] developed the bicomplex algebra and function theory. Recent works in this subject [6] find some significant applications in different fields of mathematical sciences as well as other "branches of science and technology (see, for instance [7-9] and reference therein)". An impressive body of work has been developed by a number of researchers. Among them, an important work on elementary functions of bicomplex numbers has been done by Luna-Elizaarrarás, Shapiro, Struppa and Vajiac [10]. Choi, Datta, Biswa, and Islam [11] proved some common fixed point theorems in connection with two weakly compatible mappings in bicomplex valued metric spaces. Jebril [12] proved some common fixed point theorems under rational contractions for a pair of mappings in bicomplex valued metric spaces. In 2017, Dhivya and Marudai [13] introduced the concept of complex partial metric space and suggested a plan to expand the results and proved the following common fixed point theorems under the rational expression contraction condition.

Theorem 1. Let ( $\mathfrak{W}, \preceq)$ be a partially ordered set and suppose that there exists a complex partial metric $\varrho_{c b}$ in $\mathfrak{W}$ such that $\left(\mathfrak{W}, \varrho_{c b}\right)$ is a complete complex partial metric space. Let $\Gamma, \Lambda: \mathfrak{W} \rightarrow \mathfrak{W}$ be a pair of weakly increasing mapping and suppose that, for every comparable $\sigma, \psi \in \mathfrak{W}$, we have either

$$
\varrho_{c b}(\Gamma \sigma, \Lambda \psi) \preceq a \frac{\varrho_{c b}(\sigma, \Gamma \sigma) \varrho_{c b}(\psi, \Lambda \psi)}{\varrho_{c b}(\sigma, \psi)}+b \varrho_{c b}(\sigma, \psi),
$$




$$
\begin{aligned}
& \text { for } \varrho_{c b}(\sigma, \psi) \neq 0 \text { with } a \geq 0, b \geq 0, a+b<1, \text { or } \\
& \qquad \varrho_{c b}(\Gamma \sigma, \Lambda \psi)=0 \text { if } \varrho_{c b}(\sigma, \psi)=0 .
\end{aligned}
$$

If $\Gamma$ or $\Lambda$ is continuous; then, $\Gamma$ and $\Lambda$ have a common fixed point $\propto \in \mathfrak{W}$ and $\varrho_{c b}(\alpha, \propto)=0$.

In 2019, Gunaseelan and Mishra [14] proved coupled fixed point theorems on complex partial metric space using different types of contractive conditions. In 2021, Gunaseelan, Arul Joseph, Yongji, and Zhaohui [15] proved common fixed point theorems on complex partial metric space. In 2021, Beg, Kumar Datta, and Pal [16] proved fixed point theorems on bicomplex valued metric spaces. Usually, in a metric space, self distance is zero (i.e., $\left.\varrho_{c b}(\sigma, \psi)=0\right)$, but, in partial metric space, the self distance need not be equal to zero. In this paper, inspired by Theorem 1, here we prove some common fixed point theorems on bicomplex partial metric space with an application.

\section{Preliminaries}

Throughout this paper, we denote the set of real, complex, and bicomplex numbers respectively as $\mathbb{C}_{0}, \mathbb{C}_{1}$ and $\mathbb{C}_{2}$. Segre [1] defined the bicomplex number as follows:

$$
\xi=\mathfrak{a}_{1}+\mathfrak{a}_{2} i_{1}+\mathfrak{a}_{3} i_{2}+\mathfrak{a}_{4} i_{1} i_{2}
$$

where $\mathfrak{a}_{1}, \mathfrak{a}_{2}, \mathfrak{a}_{3}, \mathfrak{a}_{4} \in \mathbb{C}_{0}$, and independent units $i_{1}, i_{2}$ are such that $i_{1}^{2}=i_{2}^{2}=-1$ and $i_{1} i_{2}=i_{2} i_{1}$, we denote that the set of bicomplex numbers $\mathbb{C}_{2}$ is defined as:

$$
\mathbb{C}_{2}=\left\{\xi: \xi=\mathfrak{a}_{1}+\mathfrak{a}_{2} i_{1}+\mathfrak{a}_{3} i_{2}+\mathfrak{a}_{4} i_{1} i_{2}, \mathfrak{a}_{1}, \mathfrak{a}_{2}, \mathfrak{a}_{3}, \mathfrak{a}_{4} \in \mathbb{C}_{0}\right\},
$$

i.e.,

$$
\mathbb{C}_{2}=\left\{\mathfrak{\xi}: \xi=\mathfrak{z}_{1}+i_{2} \mathfrak{z}_{2}, \mathfrak{z}_{1}, \mathfrak{z}_{2} \in \mathbb{C}_{1}\right\},
$$

where $\mathfrak{z}_{1}=\mathfrak{a}_{1}+\mathfrak{a}_{2} i_{1} \in \mathbb{C}_{1}$ and $\mathfrak{z}_{2}=\mathfrak{a}_{3}+\mathfrak{a}_{4} i_{1} \in \mathbb{C}_{1}$. If $\mathfrak{\xi}=\mathfrak{z}_{1}+i_{2} \mathfrak{z}_{2}$ and $\eta=\mathfrak{w}_{1}+i_{2} \mathfrak{w}_{2}$ be any two bicomplex numbers, then the sum is $\xi \pm \eta=\left(\mathfrak{z}_{1}+i_{2} \mathfrak{z}_{2}\right) \pm\left(\mathfrak{w}_{1}+i_{2} \mathfrak{w}_{2}\right)=$ $\mathfrak{z}_{1} \pm \mathfrak{w}_{1}+i_{2}\left(\mathfrak{z}_{2} \pm \mathfrak{w}_{2}\right)$ and the product is $\xi \cdot \eta=\left(\mathfrak{z}_{1}+i_{2} \mathfrak{z}_{2}\right)\left(\mathfrak{w}_{1}+i_{2} \mathfrak{w}_{2}\right)=\left(\mathfrak{z}_{1} \mathfrak{w}_{1}-\mathfrak{z}_{2} \mathfrak{w}_{2}\right)+$ $i_{2}\left(\mathfrak{z}_{1} \mathfrak{w}_{2}+\mathfrak{z}_{2} \mathfrak{w}_{1}\right)$.

Definition 1. Ref. [5] Let $\xi$ and $\eta$ be elements in $\mathbb{C}_{2}$. If $\xi^{2}=\xi$, then $\xi$ is called an idempotent element. If $\xi \neq 0, \eta \neq 0$, and $\xi \eta=0$, then $\xi$ and $\eta$ are called divisors of zero.

There are four idempotent elements in $\mathbb{C}_{2}$, they are $0,1, \mathfrak{e}_{1}=\frac{1+i_{1} i_{2}}{2}, \mathfrak{e}_{2}=\frac{1-i_{1} i_{2}}{2}$ out of which $\mathfrak{e}_{1}$ and $\mathfrak{e}_{2}$ are nontrivial such that $\mathfrak{e}_{1}+\mathfrak{e}_{2}=1$ and $\mathfrak{e}_{1} \mathfrak{e}_{2}=0$. Every bicomplex number $\mathfrak{z}_{1}+i_{2} \mathfrak{z}_{2}$ can be uniquely expressed as the combination of $\mathfrak{e}_{1}$ and $\mathfrak{e}_{2}$, namely

$$
\xi=\mathfrak{z}_{1}+i_{2} \mathfrak{z}_{2}=\left(\mathfrak{z}_{1}-i_{1} \mathfrak{z}_{2}\right) \mathfrak{e}_{1}+\left(\mathfrak{z}_{1}+i_{1} \mathfrak{z}_{2}\right) \mathfrak{e}_{2} .
$$

This representation of $\xi$ is known as the idempotent representation of bicomplex number and the complex coefficients $\xi_{1}=\left(\mathfrak{z}_{1}-i_{1} \mathfrak{z}_{2}\right)$ and $\xi_{2}=\left(\mathfrak{z}_{1}+i_{1} \mathfrak{z}_{2}\right)$ are known as idempotent components of the bicomplex number $\xi$.

An element $\tilde{\xi}=\mathfrak{z}_{1}+i_{2} \mathfrak{z}_{2} \in \mathbb{C}_{2}$ is said to be invertible if there exists another element $\eta$ in $\mathbb{C}_{2}$ such that $\xi \eta=1$ and $\eta$ is said to be inverse (multiplicative) of $\xi$. Consequently, $\xi$ is said to be the inverse (multiplicative) of $\eta$. An element which has an inverse in $\mathbb{C}_{2}$ is said to be the non-singular element of $\mathbb{C}_{2}$ and an element which does not have an inverse in $\mathbb{C}_{2}$ is said to be the singular element of $\mathbb{C}_{2}$. 
An element $\xi=\mathfrak{z}_{1}+i_{2} \mathfrak{z}_{2} \in \mathbb{C}_{2}$ is non-singular if and only if $\left|\mathfrak{z}_{1}^{2}+\mathfrak{z}_{2}^{2}\right| \neq 0$ and singular if and only if $\left|\mathfrak{z}_{1}^{2}+\mathfrak{z}_{2}^{2}\right|=0$. The inverse of $\xi$ is defined as

$$
\mathfrak{\xi}^{-1}=\eta=\frac{\mathfrak{z}-i_{2} \mathfrak{z}_{2}}{\mathfrak{z}_{1}^{2}+\mathfrak{z}_{2}^{2}} .
$$

Zero is the only element in $\mathbb{C}_{0}$ which does not have multiplicative inverse and in $\mathbb{C}_{1}$, $0=0+i 0$ is the only element which does not have a multiplicative inverse. We denote the set of singular elements of $\mathbb{C}_{0}$ and $\mathbb{C}_{1}$ by $\mathfrak{O}_{0}$ and $\mathfrak{O}_{1}$, respectively. However, there is more than one element in $\mathbb{C}_{2}$, which does not have multiplicative inverse, and we denote this set by $\mathfrak{O}_{2}$ and clearly $\mathfrak{O}_{0}=\mathfrak{O}_{1} \subset \mathfrak{O}_{2}$.

A bicomplex number $\xi=\mathfrak{a}_{1}+\mathfrak{a}_{2} i_{1}+\mathfrak{a}_{3} i_{2}+\mathfrak{a}_{4} i_{1} i_{2} \in \mathbb{C}_{2}$ is said to be degenerated if the matrix

$$
\left(\begin{array}{ll}
\mathfrak{a}_{1} & \mathfrak{a}_{2} \\
\mathfrak{a}_{3} & \mathfrak{a}_{4}
\end{array}\right)
$$

is degenerated. In that case, $\xi^{-1}$ exists, and it is also degenerated.

The norm $\|$.$\| of \mathbb{C}_{2}$ is a positive real valued function and $\|\|:. \mathbb{C}_{2} \rightarrow \mathbb{C}_{0}^{+}$is defined by

$$
\begin{aligned}
\|\mathfrak{\xi}\| & =|| \mathfrak{z}_{1}+i_{2} z_{2}||=\left\{\left|\mathfrak{z}^{2}+\right| \mathfrak{z}^{2}\right\}^{\frac{1}{2}} \\
& =\left[\frac{\left|\left(\mathfrak{z}_{1}-i_{1} \mathfrak{z}_{2}\right)\right|^{2}+\left|\left(\mathfrak{z}_{1}+i_{1} \mathfrak{z}_{2}\right)\right|^{2}}{2}\right]^{\frac{1}{2}} \\
& =\left(\mathfrak{a}_{1}^{2}+\mathfrak{a}_{2}^{2}+\mathfrak{a}_{1}^{2}+\mathfrak{a}_{3}^{2}+\mathfrak{a}_{4}^{2}\right)^{\frac{1}{2}},
\end{aligned}
$$

where $\tilde{\xi}=\mathfrak{a}_{1}+\mathfrak{a}_{2} i_{1}+\mathfrak{a}_{3} i_{2}+\mathfrak{a}_{4} i_{1} i_{2}=\mathfrak{z}_{1}+i_{2} \mathfrak{z}_{2} \in \mathbb{C}_{2}$.

The linear space $\mathbb{C}_{2}$ with respect to defined norm is a normed linear space; in addition, $\mathbb{C}_{2}$ is complete; therefore, $\mathbb{C}_{2}$ is the Banach space. If $\xi, \eta \in \mathbb{C}_{2}$, then $\|\xi \eta\| \leq \sqrt{2}\|\xi|\|\mid \eta\|$ holds instead of $\|\xi \eta\| \leq\|\xi||\| \eta \|$. Therefore, $\mathbb{C}_{2}$ is not the Banach algebra. The partial order relation $\preceq_{i_{2}}$ on $\mathbb{C}_{2}$ is defined as: Let $\mathbb{C}_{2}$ be the set of bicomplex numbers and $\xi=\mathfrak{z}_{1}+i_{2} \mathfrak{z}_{2}$, $\eta=\mathfrak{w}_{1}+i_{2} \mathfrak{w}_{2} \in \mathbb{C}_{2}$ then $\xi \preceq_{i_{2}} \eta$ if and only if $\mathfrak{z}_{1} \preceq \mathfrak{w}_{1}$ and $\mathfrak{z}_{2} \preceq \mathfrak{w}_{2}$, i.e., $\xi \preceq i_{2} \eta$ if one of the following conditions is satisfied:

(a) $\mathfrak{z}_{1}=\mathfrak{w}_{1}, \mathfrak{z}_{2}=\mathfrak{w}_{2}$,

(b) $\mathfrak{z}_{1} \prec \mathfrak{w}_{1}, \mathfrak{z}_{2}=\mathfrak{w}_{2}$,

(c) $\mathfrak{z}_{1}=\mathfrak{w}_{1}, \mathfrak{z}_{2} \prec \mathfrak{w}_{2}$, and

(d) $\mathfrak{z}_{1} \prec \mathfrak{w}_{1}, \mathfrak{z}_{2} \prec \mathfrak{w}_{2}$,

In particular, we can write $\xi \nsim_{i_{2}} \eta$ if $\xi \preceq i_{2} \eta$ and $\xi \neq \eta$ i.e., one of (b), (c), and (d) is satisfied, and we will write $\xi \prec_{i_{2}} \eta$ if only (d) is satisfied.

For any two bicomplex numbers $\xi, \eta \in \mathbb{C}_{2}$, we can verify the following:

(1) $\xi \preceq i_{i_{2}} \eta \Rightarrow\|\xi\| \leq\|\eta\|$,

(2) $\|\xi+\eta\| \leq\|\xi\|+\|\eta\|$,

(3) $\|\mathfrak{a} \xi\|=\mathfrak{a}\|\mathfrak{\xi}\|$, where $\mathfrak{a}$ is a non-negative real number,

(4) $\|\xi \eta\| \leq \sqrt{2}\|\xi||\| \eta \|$ and the equality holds only when at least one of $\xi$ and $\eta$ is degenerated,

(5) $\left\|\xi^{-1}\right\|=\|\xi\|^{-1}$ if $\xi$ is a degenerated bicomplex number with $0 \prec \xi$,

(6) $\left\|\frac{\xi}{\eta}\right\|=\frac{\|\xi\|}{\|\eta\|}$, if $\eta$ is a degenerated bicomplex number.

Now, let us recall some basic concepts and notations, which will be used in the sequel.

Definition 2. A bicomplex partial metric on a non-void set $\mathfrak{W}$ is a function $\varrho_{b c b}: \mathfrak{W} \times \mathfrak{W} \rightarrow \mathbb{C}_{2}^{+}$ such that, for all $\sigma, \psi, \vartheta \in \mathfrak{W}$ :

(i) $\quad 0 \preceq_{i_{2}} \varrho_{b c b}(\sigma, \sigma) \preceq_{i_{2}} \varrho_{b c b}(\sigma, \psi)$, (small self-distances)

(ii) $\varrho_{b c b}(\sigma, \psi)=\varrho_{b c b}(\psi, \sigma)$ (symmetry) 
(iii) $\varrho_{b c b}(\sigma, \sigma)=\varrho_{b c b}(\sigma, \psi)=\varrho_{b c b}(\psi, \psi)$ if and only if $\sigma=\psi$ (equality)

(iv) $\varrho_{b c b}(\sigma, \psi) \preceq_{i_{2}} \varrho_{b c b}(\sigma, \vartheta)+\varrho_{b c b}(\vartheta, \psi)-\varrho_{b c b}(\vartheta, \vartheta)$ (triangularity).

A bicomplex partial metric space is a pair $\left(\mathfrak{W}, \varrho_{b c b}\right)$ such that $\mathfrak{W}$ is a non-void set, and $\varrho_{b c b}$ is the bicomplex partial metric on $\mathfrak{W}$.

Example 1. Let $\mathfrak{W}=\{1,3,4,7\}$ be a set endowed with the classical bicomplex partial metric $\varrho_{b c b}(\sigma, \psi)=\left(1+i_{2}\right) \max \{\sigma, \psi\}, \forall \sigma, \psi \in \mathfrak{W}$,

\begin{tabular}{lllll}
\hline$\varrho_{c b}(\sigma, \psi)$ & 1 & 3 & 4 & 7 \\
\hline 1 & $\left(1+i_{2}\right)$ & $\left(1+i_{2}\right) 3$ & $\left(1+i_{2}\right) 4$ & $\left(1+i_{2}\right) 7$ \\
\hline 3 & $\left(1+i_{2}\right) 3$ & $\left(1+i_{2}\right) 3$ & $\left(1+i_{2}\right) 4$ & $\left(1+i_{2}\right) 7$ \\
\hline 4 & $\left(1+i_{2}\right) 4$ & $\left(1+i_{2}\right) 4$ & $\left(1+i_{2}\right) 4$ & $\left(1+i_{2}\right) 7$ \\
\hline 7 & $\left(1+i_{2}\right) 7$ & $\left(1+i_{2}\right) 7$ & $\left(1+i_{2}\right) 7$ & $\left(1+i_{2}\right) 7$ \\
\hline
\end{tabular}

Then, (i), (ii), and (iii) of Definition 2 are obvious for the function $\varrho_{b c b}$. Let $\sigma=1, \psi=3$, $\vartheta=4 \in \mathfrak{W}$ be arbitrary.

Now,

$$
\begin{aligned}
\varrho_{b c b}(1,3)=\left(1+i_{2}\right) 3 & \preceq_{i_{2}} 4\left(1+i_{2}\right)+4\left(1+i_{2}\right)-4\left(1+i_{2}\right) \\
& =\varrho_{b c b}(1,4)+\varrho_{b c b}(4,3)-\varrho_{b c b}(4,4) .
\end{aligned}
$$

Therefore, $\varrho_{b c b}(\sigma, \psi) \preceq_{i_{2}} \varrho_{b c b}(\sigma, \vartheta)+\varrho_{b c b}(\vartheta, \psi)-\varrho_{b c b}(\vartheta, \vartheta)$. Hence, $\left(\mathfrak{W}, \varrho_{b c b}\right)$ is a bicomplex partial metric space.

For the bicomplex partial metric space $\varrho_{b c b}$ on $\mathfrak{W}$, the function $\mathfrak{d}_{\varrho_{b c b}}: \mathfrak{W} \times \mathfrak{W} \rightarrow \mathbb{C}_{2}^{+}$ given by $\mathfrak{d}_{\varrho_{b c b}}=2 \varrho_{b c b}(\sigma, \psi)-\varrho_{b c b}(\sigma, \sigma)-\varrho_{b c b}(\psi, \psi)$ is a usual metric on $\mathfrak{W}$. Each bicomplex partial metric $\varrho_{b c b}$ on $\mathfrak{W}$ generates a topology $\tau_{\varrho_{b c b}}$ on $\mathfrak{W}$ with the base family of open $\varrho_{b c b}$-balls $\left\{\mathfrak{B}_{\varrho_{b c b}}(\sigma, \epsilon): \sigma \in \mathfrak{W}, \epsilon \succ_{i_{2}} 0\right\}$, where $\mathfrak{B}_{\varrho_{b c b}}(\sigma, \epsilon)=\left\{\psi \in \mathfrak{W}: \varrho_{b c b}(\sigma, \psi) \prec_{i_{2}}\right.$ $\left.\varrho_{b c b}(\sigma, \sigma)+\epsilon\right\}$ for all $\sigma \in \mathfrak{W}$ and $0 \prec i_{2} \in \in \mathbb{C}_{2}^{+}$.

A bicomplex valued metric space is a bicomplex partial metric space. However, a bicomplex partial metric space need not be a bicomplex valued metric space. The above Example 1 illustrates such a bicomplex partial metric space.

Note that self distance need not be zero, for example $\varrho_{b c b}(1,1)=1+i_{2} \neq 0$. Now, the metric induced by $\varrho_{b c b}$ is as follows: $\mathfrak{d}_{\varrho_{b c b}}=2 \varrho_{b c b}(\sigma, \psi)-\varrho_{b c b}(\sigma, \sigma)-\varrho_{b c b}(\psi, \psi)$; without loss of generality, suppose $\sigma \geq \psi$ then $\mathfrak{d}_{\varrho_{b c b}}=2\left\{\max \{\sigma, \psi\}+i_{2} \max \{\sigma, \psi\}\right\}-\left\{\sigma+i_{2} \sigma\right\}-$ $\left\{\psi+i_{2} \psi\right\}$. Therefore, $\mathfrak{d}_{\varrho_{b c b}}(\sigma, \psi)=|\sigma-\psi|+i_{2}|\sigma-\psi|$.

Theorem 2. Let $\left(\mathfrak{W}, \varrho_{b c b}\right)$ be a bicomplex partial metric space, then $\left(\mathfrak{W}, \varrho_{b c b}\right)$ is $\mathcal{T}_{0}$.

Proof. Supposing $\sigma, \psi \in \mathfrak{W}$ and $\sigma \neq \psi$, from condition (i) and (iii) in Definition 2, we get

$$
\begin{gathered}
\varrho_{b c b}(\sigma, \sigma) \prec_{i_{2}} \varrho_{b c b}(\sigma, \psi) \\
\text { OR } \\
\varrho_{b c b}(\psi, \psi) \prec_{i_{2}} \varrho_{b c b}(\sigma, \psi) .
\end{gathered}
$$

Suppose that $\varrho_{b c b}(\sigma, \sigma) \prec_{i_{2}} \varrho_{b c b}(\sigma, \psi)$. Then, we have $0 \prec_{i_{2}} \varrho_{b c b}(\sigma, \psi)-\varrho_{b c b}(\sigma, \sigma)$. Now, let $\mathfrak{r} \in \mathbb{C}_{2}^{+}$such that $0 \prec_{i_{2}} \mathfrak{r} \prec_{i_{2}} \varrho_{b c b}(\sigma, \psi)-\varrho_{b c b}(\sigma, \sigma)$. Therefore, $\sigma \in \mathfrak{B}_{\varrho_{b c b}}(\sigma, \mathfrak{r})$ and $\psi \notin \mathfrak{B}_{\varrho_{b c b}}(\sigma, \mathfrak{r})$. Hence, $\left(\mathfrak{W}, \varrho_{b c b}\right)$ is $\mathcal{T}_{0}$.

Definition 3. Let $\left(\mathfrak{W}, \varrho_{b c b}\right)$ be a bicomplex partial metric space. A sequence $\left\{\sigma_{\alpha}\right\}$ in $\mathfrak{W}$ is said to be a convergent and converges to $\sigma \in \mathfrak{W}$ if, for every $0 \prec_{i_{2}} \in \in \mathbb{C}_{2}^{+}$, there exists $\mathfrak{N} \in \mathbb{N}$ such that $\sigma_{\alpha} \in \mathfrak{B}_{\varrho_{b c b}}(\sigma, \epsilon)$ for all $\alpha \geq \mathfrak{N}$, and it is denoted by $\lim _{\alpha \rightarrow \infty} \sigma_{\alpha}=\sigma$. 
Lemma 1. Let $\left(\mathfrak{W}, \varrho_{b c b}\right)$ be a bicomplex partial metric space. A sequence $\left\{\sigma_{\alpha}\right\} \in \mathfrak{W}$ converges to $\sigma \in \mathfrak{W}$ iff $\varrho_{b c b}(\sigma, \sigma)=\lim _{\alpha \rightarrow \infty} \varrho_{b c b}\left(\sigma, \sigma_{\alpha}\right)$.

Proof. Assume that $\left\{\sigma_{n}\right\}$ converges to $\sigma$. Let $\epsilon>0$ be any real number. Suppose

$$
\mathfrak{r}=\frac{\epsilon}{2}+i_{1} \frac{\epsilon}{2}+i_{2} \frac{\epsilon}{2}+i_{1} i_{2} \frac{\epsilon}{2} .
$$

Then, $0 \prec_{i_{2}} \mathfrak{r} \in \mathbb{C}_{2}^{+}$and, for this $\mathfrak{r}$, there is a natural number $\mathfrak{N} \in \mathbb{N}$ such that $\sigma_{\alpha} \in \mathfrak{B}_{\varrho_{b c b}}(\sigma, \mathfrak{r})$ for all $\alpha \geq \mathfrak{N}$ i.e., $\varrho_{b c b}\left(\sigma_{\alpha}, \sigma\right) \prec_{i_{2}} \mathfrak{r}+\varrho_{b c b}(\sigma, \sigma)$. Therefore,

$$
\left\|\varrho_{b c b}\left(\sigma_{\alpha}, \sigma\right)-\varrho_{b c b}(\sigma, \sigma)\right\|<\epsilon \text { for all } \alpha \geq \mathfrak{N}
$$

Therefore, $\varrho_{b c b}\left(\sigma_{\alpha}, \sigma\right) \rightarrow \varrho_{b c b}(\sigma, \sigma)$ as $\alpha \rightarrow \infty$.

Conversely, assume that $\varrho_{b c b}\left(\sigma_{\alpha}, \sigma\right) \rightarrow \varrho_{b c b}(\sigma, \sigma)$ as $\alpha \rightarrow \infty$. Then, for each $0 \prec_{i_{2}} \mathfrak{r} \in$ $\mathbb{C}_{2}^{+}$, there exists a real $\epsilon>0$ such that, for all $\xi \in \mathbb{C}_{2}^{+}$,

$$
\|\xi\|<\epsilon \Rightarrow \xi \prec_{i_{2}} \mathfrak{r} .
$$

Then, for this $\epsilon>0$, there exists $\mathfrak{N} \in \mathbb{N}$ such that

$$
\left\|\varrho_{b c b}\left(\sigma_{\alpha}, \sigma\right)-\varrho_{b c b}(\sigma, \sigma)\right\|<\epsilon \text { for all } \alpha \geq \mathfrak{N} .
$$

Therefore,

$$
\varrho_{b c b}\left(\sigma_{\alpha}, \sigma\right) \prec_{i_{2}} \mathfrak{r}+\varrho_{b c b}(\sigma, \sigma) \text { for all } \alpha \geq \mathfrak{N} \text {. }
$$

Hence, $\left\{\sigma_{\alpha}\right\}$ converges to a point $\sigma$.

Definition 4. Let $\left(\mathfrak{W}, \varrho_{b c b}\right)$ be a bicomplex partial metric space. A sequence $\left\{\sigma_{\alpha}\right\}$ in $\mathfrak{W}$ is said to be a Cauchy sequence in $\left(\mathfrak{W}, \varrho_{b c b}\right)$ if, for any $\epsilon>0$, there exist $\mathfrak{a} \in \mathbb{C}_{2}^{+}$and $\mathfrak{N} \in \mathbb{N}$ such that $\left\|\varrho_{b c b}\left(\sigma_{\beta}, \sigma_{\alpha}\right)-\mathfrak{a}\right\|<\epsilon$ for all $\alpha, \beta \in \mathbb{N}$ and $\alpha, \beta \geq \mathfrak{N}$.

Definition 5. Let $\left(\mathfrak{W}, \varrho_{b c b}\right)$ be a bicomplex partial metric space. Let $\left\{\sigma_{\alpha}\right\}$ be any sequence in W. Then,

(i) If every Cauchy sequence in $\mathfrak{W}$ is convergent in $\mathfrak{W}$, then $\left(\mathfrak{W}, \varrho_{b c b}\right)$ is said to be a complete bicomplex partial metric space.

(ii) A mapping $\Gamma: \mathfrak{W} \rightarrow \mathfrak{W}$ is said to be continuous at $\sigma_{0} \in \mathfrak{W}$ if, for every $\epsilon>0$, there exists $\delta>0$ such that $\Gamma\left(\mathfrak{B}_{\varrho_{b c b}}\left(\sigma_{0}, \delta\right)\right) \subset \mathfrak{B}_{Q_{b c b}}\left(\Gamma\left(\sigma_{0}, \epsilon\right)\right)$.

Lemma 2. Let $\left(\mathfrak{W}, \varrho_{b c b}\right)$ be a bicomplex partial metric space and $\left\{\sigma_{\alpha}\right\}$ be a sequence in $\mathfrak{W}$. Then, $\left\{\sigma_{\alpha}\right\}$ is a Cauchy sequence in $\mathfrak{W}$ iff $\lim _{\alpha \rightarrow \infty} \varrho_{b c b}\left(\sigma_{\alpha}, \sigma_{\beta}\right)=\varrho_{b c b}(\sigma, \sigma)$.

Proof. Assume that $\left\{\sigma_{\alpha}\right\}$ is a Cauchy sequence in $\mathfrak{W}$. Let $\epsilon>0$ be any real number. Suppose

$$
\mathfrak{r}=\frac{\epsilon}{2}+i_{1} \frac{\epsilon}{2}+i_{2} \frac{\epsilon}{2}+i_{1} i_{2} \frac{\epsilon}{2}
$$

Then, $0 \prec_{i_{2}} \mathfrak{r} \in \mathbb{C}_{2}^{+}$and, for this $\mathfrak{r}$, there is a natural number $\mathfrak{N} \in \mathbb{N}$ such that $\sigma_{\alpha} \in \mathfrak{B}_{\varrho_{b c b}}\left(\sigma_{\beta}, \mathfrak{r}\right)$ for all $\alpha, \beta \geq \mathfrak{N}$ i.e., $\varrho_{b c b}\left(\sigma_{\alpha}, \sigma_{\beta}\right) \prec_{i_{2}} \mathfrak{r}+\varrho_{b c b}(\sigma, \sigma)$. Therefore,

$$
\left\|\varrho_{b c b}\left(\sigma_{\alpha}, \sigma_{\beta}\right)-\varrho_{b c b}(\sigma, \sigma)\right\|<\epsilon \text { for all } \alpha, \beta \geq \mathfrak{N}
$$

Therefore, $\varrho_{b c b}\left(\sigma_{\alpha}, \sigma_{\beta}\right) \rightarrow \varrho_{b c b}(\sigma, \sigma)$ as $\alpha, \beta \rightarrow \infty$. 
Conversely, assume that $\varrho_{b c b}\left(\sigma_{\alpha}, \sigma_{\beta}\right) \rightarrow \varrho_{b c b}(\sigma, \sigma)$ as $\alpha, \beta \rightarrow \infty$. Then, for each $0 \prec_{i_{2}}$ $\mathfrak{r} \in \mathbb{C}_{2}^{+}$, there exists a real $\epsilon>0$ such that, for all $\xi \in \mathbb{C}_{2}^{+}$,

$$
\|\xi\|<\epsilon \Rightarrow \xi \prec_{i_{2}} \text { r. }
$$

Then, for this $\epsilon>0$, there exists $\mathfrak{N} \in \mathbb{N}$ such that

$$
\left\|\varrho_{b c b}\left(\sigma_{\alpha}, \sigma_{\beta}\right)-\varrho_{b c b}(\sigma, \sigma)\right\|<\epsilon \text { for all } \alpha, \beta \geq \mathfrak{N} .
$$

Therefore,

$$
\varrho_{b c b}\left(\sigma_{\alpha}, \sigma_{\beta}\right) \prec_{i_{2}} \mathfrak{r}+\varrho_{b c b}(\sigma, \sigma) \text { for all } \alpha, \beta \geq \mathfrak{N} .
$$

Hence, $\left\{\sigma_{\alpha}\right\}$ is a Cauchy sequence.

Definition 6. Let $\Gamma$ and $\Lambda$ be self mappings of non-void set $\mathfrak{W}$. A point $\sigma \in \mathfrak{W}$ is called a common fixed point of $\Gamma$ and $\Lambda$ if $\sigma=\Gamma \sigma=\Lambda \sigma$.

\section{Main Results}

Theorem 3. Let $\left(\mathfrak{W}, \varrho_{b c b}\right)$ be a complete bicomplex partial metric space and $\Gamma, \Lambda: \mathfrak{W} \rightarrow \mathfrak{W}$ be two continuous mappings such that

$$
\begin{aligned}
\varrho_{b c b}(\Gamma \sigma, \Lambda \psi) \preceq & i_{2} \curlywedge \max \left\{\varrho_{b c b}(\sigma, \psi), \varrho_{b c b}(\sigma, \Gamma \sigma), \varrho_{b c b}(\psi, \Lambda \psi),\right. \\
& \left.\frac{1}{2}\left(\varrho_{b c b}(\sigma, \Lambda \psi)+\varrho_{b c b}(\psi, \Gamma \sigma)\right)\right\},
\end{aligned}
$$

for all $\sigma, \psi \in \mathfrak{W}$, where $0 \leq \curlywedge<1$. Then, the pair $(\Gamma, \Lambda)$ has a unique common fixed point and $\varrho_{b c b}\left(\sigma^{*}, \sigma^{*}\right)=0$.

Proof. Let $\sigma_{0}$ be arbitrary point in $\mathfrak{W}$ and define a sequence $\left\{\sigma_{\alpha}\right\}$ as follows:

$$
\sigma_{2 \alpha+1}=\Gamma \sigma_{2 \alpha} \text { and } \sigma_{2 \alpha+2}=\Lambda \sigma_{2 \alpha+1}, \alpha=0,1,2, \ldots
$$

Then, by (1) and (2), we obtain

$$
\begin{aligned}
\varrho_{b c b}\left(\sigma_{2 \alpha+1}, \sigma_{2 \alpha+2}\right)= & \varrho_{b c b}\left(\Gamma \sigma_{2 \alpha}, \Lambda \sigma_{2 \alpha+1}\right) \\
\preceq_{i_{2}} \curlywedge \max \left\{\varrho_{b c b}\left(\sigma_{2 \alpha}, \sigma_{2 \alpha+1}\right), \varrho_{b c b}\left(\sigma_{2 \alpha}, \Gamma \sigma_{2 \alpha}\right), \varrho_{b c b}\left(\sigma_{2 \alpha+1}, \Lambda \sigma_{2 \alpha+1}\right),\right. & \\
& \left.\frac{1}{2}\left(\varrho_{b c b}\left(\sigma_{2 \alpha}, \Lambda \sigma_{2 \alpha+1}\right)+\varrho_{b c b}\left(\sigma_{2 \alpha+1}, \Gamma \sigma_{2 \alpha}\right)\right)\right\} \\
\preceq_{i_{2}} \curlywedge \max \left\{\varrho_{b c b}\left(\sigma_{2 \alpha}, \sigma_{2 \alpha+1}\right), \varrho_{b c b}\left(\sigma_{2 \alpha}, \sigma_{2 \alpha+1}\right), \varrho_{b c b}\left(\sigma_{2 \alpha+1}, \sigma_{2 \alpha+2}\right),\right. & \\
& \left.\frac{1}{2}\left(\varrho_{b c b}\left(\sigma_{2 \alpha}, \sigma_{2 \alpha+2}\right)+\varrho_{b c b}\left(\sigma_{2 \alpha+1}, \sigma_{2 \alpha+1}\right)\right)\right\} \\
\preceq_{i_{2}} \curlywedge \max \left\{\varrho_{b c b}\left(\sigma_{2 \alpha}, \sigma_{2 \alpha+1}\right), \varrho_{b c b}\left(\sigma_{2 \alpha+1}, \sigma_{2 \alpha+2}\right),\right. & \\
& \frac{1}{2}\left(\varrho_{b c b}\left(\sigma_{2 \alpha}, \sigma_{2 \alpha+1}\right)+\varrho_{b c b}\left(\sigma_{2 \alpha+1}, \sigma_{2 \alpha+2}\right)-\varrho_{b c b}\left(\sigma_{2 \alpha+1}, \sigma_{2 \alpha+1}\right)\right. \\
& \left.\left.+\varrho_{b c b}\left(\sigma_{2 \alpha+1}, \sigma_{2 \alpha+1}\right)\right)\right\} \\
= & \curlywedge \max \left\{\varrho_{b c b}\left(\sigma_{2 \alpha}, \sigma_{2 \alpha+1}\right), \varrho_{b c b}\left(\sigma_{2 \alpha+1}, \sigma_{2 \alpha+2}\right),\right. \\
& \left.\frac{1}{2}\left(\varrho_{b c b}\left(\sigma_{2 \alpha}, \sigma_{2 \alpha+1}\right)+\varrho_{b c b}\left(\sigma_{2 \alpha+1}, \sigma_{2 \alpha+2}\right)\right)\right\} .
\end{aligned}
$$

Case I: If $\max \left\{\varrho_{b c b}\left(\sigma_{2 \alpha}, \sigma_{2 \alpha+1}\right), \varrho_{b c b}\left(\sigma_{2 \alpha+1}, \sigma_{2 \alpha+2}\right)\right.$, $\left.\frac{1}{2}\left(\varrho_{b c b}\left(\sigma_{2 \alpha}, \sigma_{2 \alpha+1}\right)+\varrho_{b c b}\left(\sigma_{2 \alpha+1}, \sigma_{2 \alpha+2}\right)\right)\right\}=\varrho_{b c b}\left(\sigma_{2 \alpha+1}, \sigma_{2 \alpha+2}\right)$, then we have

$$
\varrho_{b c b}\left(\sigma_{2 \alpha+1}, \sigma_{2 \alpha+2}\right) \preceq_{i_{2}} \curlywedge \varrho_{b c b}\left(\sigma_{2 \alpha+1}, \sigma_{2 \alpha+2}\right) .
$$


This implies $\curlywedge \geq 1$, which is a contradiction.

Case II: If $\max \left\{\varrho_{b c b}\left(\sigma_{2 \alpha}, \sigma_{2 \alpha+1}\right), \varrho_{b c b}\left(\sigma_{2 \alpha+1}, \sigma_{2 \alpha+2}\right), \frac{1}{2}\left(\varrho_{b c b}\left(\sigma_{2 \alpha}, \sigma_{2 \alpha+1}\right)+\varrho_{b c b}\left(\sigma_{2 \alpha+1}, \sigma_{2 \alpha+2}\right)\right)\right\}$ $=\varrho_{b c b}\left(\sigma_{2 \alpha}, \sigma_{2 \alpha+1}\right)$, then we have

$$
\varrho_{b c b}\left(\sigma_{2 \alpha+1}, \sigma_{2 \alpha+2}\right) \preceq_{i_{2}} \curlywedge \varrho_{b c b}\left(\sigma_{2 \alpha}, \sigma_{2 \alpha+1}\right) .
$$

From the next step, we have

$$
\begin{aligned}
\varrho_{b c b}\left(\sigma_{2 \alpha+2}, \sigma_{2 \alpha+3}\right) \preceq_{i_{2}} \curlywedge \max \left\{\varrho_{b c b}\left(\sigma_{2 \alpha+1}, \sigma_{2 \alpha+2}\right), \varrho_{b c b}\left(\sigma_{2 \alpha+2}, \sigma_{2 \alpha+3}\right),\right. & \\
& \left.\frac{1}{2}\left(\varrho_{b c b}\left(\sigma_{2 \alpha+1}, \sigma_{2 \alpha+2}\right)+\varrho_{b c b}\left(\sigma_{2 \alpha+2}, \sigma_{2 \alpha+3}\right)\right)\right\} .
\end{aligned}
$$

The following three cases arise, and we have

\section{Case IIa:}

$$
\varrho_{b c b}\left(\sigma_{2 \alpha+2}, \sigma_{2 \alpha+3}\right) \preceq_{i_{2}} \curlywedge \varrho_{b c b}\left(\sigma_{2 \alpha+2}, \sigma_{2 \alpha+3}\right),
$$

which implies $\curlywedge \geq 1$ and is a contradiction.

\section{Case IIb:}

$$
\varrho_{b c b}\left(\sigma_{2 \alpha+2}, \sigma_{2 \alpha+3}\right) \preceq_{i_{2}} \curlywedge \varrho_{b c b}\left(\sigma_{2 \alpha+1}, \sigma_{2 \alpha+2}\right) .
$$

From (3) and (4), $\forall \alpha=0,1,2, \ldots$, we get

$$
\varrho_{b c b}\left(\sigma_{\alpha+1}, \sigma_{\alpha+2}\right) \preceq_{i_{2}} \curlywedge \varrho_{b c b}\left(\sigma_{\alpha}, \sigma_{\alpha+1}\right) \preceq_{i_{2}} \ldots \preceq_{i_{2}} \curlywedge^{\alpha+1} \varrho_{b c b}\left(\sigma_{0}, \sigma_{1}\right) .
$$

For $\beta, \alpha \in \mathbb{N}$, with $\beta>\alpha$, we have

$$
\begin{aligned}
& \varrho_{b c b}\left(\sigma_{\alpha}, \sigma_{\beta}\right) \preceq_{i_{2}} \varrho_{b c b}\left(\sigma_{\alpha}, \sigma_{\alpha+1}\right)+\varrho_{b c b}\left(\sigma_{\alpha+1}, \sigma_{\beta}\right)-\varrho_{b c b}\left(\sigma_{\alpha+1}, \sigma_{\alpha+1}\right) \\
& \preceq_{i_{2}} \varrho_{b c b}\left(\sigma_{\alpha}, \sigma_{\alpha+1}\right)+\varrho_{b c b}\left(\sigma_{\alpha+1}, \sigma_{\beta}\right) \\
& \preceq_{i_{2}} \varrho_{b c b}\left(\sigma_{\alpha}, \sigma_{\alpha+1}\right)+\varrho_{b c b}\left(\sigma_{\alpha+1}, \sigma_{\alpha+2}\right)+\varrho_{b c b}\left(\sigma_{\alpha+2}, \sigma_{\beta}\right) \\
&-\varrho_{b c b}\left(\sigma_{\alpha+2}, \sigma_{\alpha+2}\right) \\
& \preceq_{i_{2}} \varrho_{b c b}\left(\sigma_{\alpha}, \sigma_{\alpha+1}\right)+\varrho_{b c b}\left(\sigma_{\alpha+1}, \sigma_{\alpha+2}\right)+\varrho_{b c b}\left(\sigma_{\alpha+2}, \sigma_{\beta}\right) \\
& \preceq_{i_{2}} \varrho_{b c b}\left(\sigma_{\alpha}, \sigma_{\alpha+1}\right)+\varrho_{b c b}\left(\sigma_{\alpha+1}, \sigma_{\alpha+2}\right)+\varrho_{b c b}\left(\sigma_{\alpha+2}, \sigma_{\alpha+3}\right) \\
&+\ldots+\varrho_{b c b}\left(\sigma_{\beta-2}, \sigma_{\beta-1}\right)+\varrho_{b c b}\left(\sigma_{\beta-1}, \sigma_{\beta}\right) .
\end{aligned}
$$

Moreover, by using (4), we get

$$
\begin{aligned}
\varrho_{b c b}\left(\sigma_{\alpha}, \sigma_{\beta}\right) \preceq & i_{2} \curlywedge^{\alpha} \varrho_{b c b}\left(\sigma_{0}, \sigma_{1}\right)+\curlywedge^{\alpha+1} \varrho_{b c b}\left(\sigma_{0}, \sigma_{1}\right)+\curlywedge^{\alpha+2} \varrho_{b c b}\left(\sigma_{0}, \sigma_{1}\right) \\
& +\ldots+\curlywedge^{\beta-2} \varrho_{b c b}\left(\sigma_{0}, \sigma_{1}\right)+\curlywedge^{\beta-1} \varrho_{b c b}\left(\sigma_{0}, \sigma_{1}\right) \\
= & \sum_{i=1}^{\beta-\alpha} \curlywedge^{i+\alpha-1} \varrho_{b c b}\left(\sigma_{0}, \sigma_{1}\right) .
\end{aligned}
$$

Therefore,

$$
\begin{aligned}
\left\|\varrho_{b c b}\left(\sigma_{\alpha}, \sigma_{\beta}\right)\right\| & \leq \sum_{i=1}^{\beta-\alpha} \curlywedge^{i+\alpha-1}\left\|\varrho_{b c b}\left(\sigma_{0}, \sigma_{1}\right)\right\|=\sum_{t=\alpha}^{\beta-1} \curlywedge^{t}\left\|\varrho_{b c b}\left(\sigma_{0}, \sigma_{1}\right)\right\| \\
& \leq \sum_{i=\alpha}^{\infty}\left\|\varrho_{b c b}\left(\sigma_{0}, \sigma_{1}\right)\right\| \\
& =\frac{\curlywedge^{\alpha}}{1-\curlywedge}\left\|\varrho_{b c b}\left(\sigma_{0}, \sigma_{1}\right)\right\| .
\end{aligned}
$$


Then, we have

$$
\left\|\varrho_{b c b}\left(\sigma_{\alpha}, \sigma_{\beta}\right)\right\| \leq \frac{\curlywedge^{\alpha}}{1-\curlywedge}\left\|\varrho_{b c b}\left(\sigma_{0}, \sigma_{1}\right)\right\| \rightarrow 0 \quad \text { as } \quad \alpha \rightarrow \infty .
$$

Case IIc:

Hence, $\left\{\sigma_{\alpha}\right\}$ is a Cauchy sequence in $\mathfrak{W}$.

$$
\varrho_{b c b}\left(\sigma_{2 \alpha+2}, \sigma_{2 \alpha+3}\right) \preceq_{i_{2}} \curlywedge \frac{1}{2}\left(\varrho_{b c b}\left(\sigma_{2 \alpha+1}, \sigma_{2 \alpha+2}\right)+\varrho_{b c b}\left(\sigma_{2 \alpha+2}, \sigma_{2 \alpha+3}\right)\right) .
$$

This implies that

$$
\varrho_{b c b}\left(\sigma_{2 \alpha+2}, \sigma_{2 \alpha+3}\right) \preceq_{i_{2}} \frac{\curlywedge}{(2-\curlywedge)} \varrho_{b c b}\left(\sigma_{2 \alpha+1}, \sigma_{2 \alpha+2}\right) .
$$

Since $a:=\frac{\curlywedge}{2-\curlywedge}<1$, we get $\varrho_{b c b}\left(\sigma_{\alpha+1}, \sigma_{\alpha+2}\right) \preceq_{i_{2}} a \varrho_{b c b}\left(\sigma_{\alpha}, \sigma_{\alpha+1}\right)$. Using Case IIb, we get that $\left\{\sigma_{\alpha}\right\}_{\alpha \in \mathbb{N}}$ is a Cauchy sequence in $\mathfrak{W}$.

\section{Case III:}

If $\max \left\{\varrho_{b c b}\left(\sigma_{2 \alpha}, \sigma_{2 \alpha+1}\right), \varrho_{b c b}\left(\sigma_{2 \alpha+1}, \sigma_{2 \alpha+2}\right), \frac{1}{2}\left(\varrho_{b c b}\left(\sigma_{2 \alpha}, \sigma_{2 \alpha+1}\right)+\varrho_{b c b}\left(\sigma_{2 \alpha+1}, \sigma_{2 \alpha+2}\right)\right)\right\}=$ $\frac{1}{2}\left(\varrho_{b c b}\left(\sigma_{2 \alpha}, \sigma_{2 \alpha+1}\right)+\varrho_{b c b}\left(\sigma_{2 \alpha+1}, \sigma_{2 \alpha+2}\right)\right)$. Then, we have

$$
\varrho_{b c b}\left(\sigma_{2 \alpha+1}, \sigma_{2 \alpha+2}\right) \preceq_{i_{2}} \frac{\curlywedge}{2}\left(\varrho_{b c b}\left(\sigma_{2 \alpha}, \sigma_{2 \alpha+1}\right)+\varrho_{b c b}\left(\sigma_{2 \alpha+1}, \sigma_{2 \alpha+2}\right)\right) .
$$

Hence,

$$
\varrho_{b c b}\left(\sigma_{2 \alpha+1}, \sigma_{2 \alpha+2}\right) \preceq_{i_{2}} \frac{\curlywedge}{2-\curlywedge} \varrho_{b c b}\left(\sigma_{2 \alpha}, \sigma_{2 \alpha+1}\right) .
$$

For the next step, we have

$$
\begin{aligned}
& \varrho_{b c b}\left(\sigma_{2 \alpha+2}, \sigma_{2 \alpha+3}\right) \preceq_{i_{2}} \curlywedge \max \left\{\varrho_{b c b}\left(\sigma_{2 \alpha+1}, \sigma_{2 \alpha+2}\right), \varrho_{b c b}\left(\sigma_{2 \alpha+2}, \sigma_{2 \alpha+3}\right),\right. \\
&\left.\frac{1}{2}\left(\varrho_{b c b}\left(\sigma_{2 \alpha+1}, \sigma_{2 \alpha+2}\right)+\varrho_{b c b}\left(\sigma_{2 \alpha+2}, \sigma_{2 \alpha+3}\right)\right)\right\} .
\end{aligned}
$$

Then, we have the following three cases:

\section{Case IIIa:}

$$
\varrho_{b c b}\left(\sigma_{2 \alpha+2}, \sigma_{2 \alpha+3}\right) \preceq_{i_{2}} \curlywedge \varrho_{b c b}\left(\sigma_{2 \alpha+2}, \sigma_{2 \alpha+3}\right),
$$

which implies $\curlywedge \geq 1$, which is a contradiction.

\section{Case IIIb:}

$$
\varrho_{b c b}\left(\sigma_{2 \alpha+2}, \sigma_{2 \alpha+3}\right) \preceq_{i_{2}} \curlywedge \varrho_{b c b}\left(\sigma_{2 \alpha+1}, \sigma_{2 \alpha+2}\right) .
$$

Then, by (6) and (7), we get $\varrho_{b c b}\left(\sigma_{\alpha+1}, \sigma_{\alpha+2}\right) \preceq_{i_{2}} \gamma \varrho_{b c b}\left(\sigma_{\alpha}, \sigma_{\alpha+1}\right)$, where $\gamma=\max \left\{\curlywedge, \frac{\curlywedge}{2-\curlywedge}\right\}<1$. Hence, $\left\{\sigma_{\alpha}\right\}_{\alpha \in \mathbb{N}}$ is a Cauchy sequence in $\mathfrak{W}$.

Case IIIc:

$$
\varrho_{b c b}\left(\sigma_{2 \alpha+2}, \sigma_{2 \alpha+3}\right) \preceq_{i_{2}} \frac{1}{2}\left(\varrho_{b c b}\left(\sigma_{2 \alpha+1}, \sigma_{2 \alpha+2}\right)+\varrho_{b c b}\left(\sigma_{2 \alpha+2}, \sigma_{2 \alpha+3}\right)\right) .
$$

Hence, we obtain

$$
\varrho_{b c b}\left(\sigma_{2 \alpha+2}, \sigma_{2 \alpha+3}\right) \preceq_{i_{2}} \frac{\curlywedge}{(2-\curlywedge)} \varrho_{b c b}\left(\sigma_{2 \alpha+1}, \sigma_{2 \alpha+2}\right) .
$$


Using (6) and (8) yield

$$
\varrho_{b c b}\left(\sigma_{\alpha+1}, \sigma_{\alpha+2}\right) \preceq_{i_{2}} \prec \varrho_{b c b}\left(\sigma_{\alpha}, \sigma_{\alpha+1}\right),
$$

where $0 \leq \imath=\frac{\curlywedge}{2-\ell}<1$.

Then, $\forall \alpha=0,1,2, \ldots$, we get

$$
\varrho_{b c b}\left(\sigma_{\alpha+1}, \sigma_{\alpha+2}\right) \preceq_{i_{2}} \prec \varrho_{b c b}\left(\sigma_{\alpha}, \sigma_{\alpha+1}\right) \preceq_{i_{2}} \ldots \preceq_{i_{2}} 2^{\alpha+1} \varrho_{b c b}\left(\sigma_{0}, \sigma_{1}\right) .
$$

For $\beta, \alpha \in \mathbb{N}$, with $\beta>\alpha$, we have

$$
\begin{aligned}
& \varrho_{b c b}\left(\sigma_{\alpha}, \sigma_{\beta}\right) \varliminf_{i_{2}} \varrho_{b c b}\left(\sigma_{\alpha}, \sigma_{\alpha+1}\right)+\varrho_{b c b}\left(\sigma_{\alpha+1}, \sigma_{\beta}\right)-\varrho_{b c b}\left(\sigma_{\alpha+1}, \sigma_{\alpha+1}\right) \\
& \preceq_{i_{2}} \varrho_{b c b}\left(\sigma_{\alpha}, \sigma_{\alpha+1}\right)+\varrho_{b c b}\left(\sigma_{\alpha+1}, \sigma_{\beta}\right) \\
& \preceq_{i_{2}} \varrho_{b c b}\left(\sigma_{\alpha}, \sigma_{\alpha+1}\right)+\varrho_{b c b}\left(\sigma_{\alpha+1}, \sigma_{\alpha+2}\right)+\varrho_{b c b}\left(\sigma_{\alpha+2}, \sigma_{\beta}\right) \\
&-\varrho_{b c b}\left(\sigma_{\alpha+2}, \sigma_{\alpha+2}\right) \\
& \preceq_{i_{2}} \varrho_{b c b}\left(\sigma_{\alpha}, \sigma_{\alpha+1}\right)+\varrho_{b c b}\left(\sigma_{\alpha+1}, \sigma_{\alpha+2}\right)+\varrho_{b c b}\left(\sigma_{\alpha+2}, \sigma_{\beta}\right) \\
& \preceq_{i_{2}} \varrho_{b c b}\left(\sigma_{\alpha}, \sigma_{\alpha+1}\right)+\varrho_{b c b}\left(\sigma_{\alpha+1}, \sigma_{\alpha+2}\right)+\varrho_{b c b}\left(\sigma_{\alpha+2}, \sigma_{\alpha+3}\right) \\
&\left.+\ldots+\varrho_{b c b}\left(\sigma_{\beta-2}, \sigma_{\beta-1}\right)\right)+\varrho_{b c b}\left(\sigma_{\beta-1}, \sigma_{\beta}\right) .
\end{aligned}
$$

Using (9), we get

$$
\begin{aligned}
\varrho_{b c b}\left(\sigma_{\alpha}, \sigma_{\beta}\right) \preceq & i_{2} 2^{\alpha} \varrho_{b c b}\left(\sigma_{0}, \sigma_{1}\right)+2^{\alpha+1} \varrho_{b c b}\left(\sigma_{0}, \sigma_{1}\right)+2^{\alpha+2} \varrho_{b c b}\left(\sigma_{0}, \sigma_{1}\right) \\
& +\ldots+2^{\beta-2} \varrho_{b c b}\left(\sigma_{0}, \sigma_{1}\right)+2^{\beta-1} \varrho_{b c b}\left(\sigma_{0}, \sigma_{1}\right) \\
= & \sum_{i=1}^{\beta-\alpha} 2^{i+\alpha-1} \varrho_{b c b}\left(\sigma_{0}, \sigma_{1}\right) .
\end{aligned}
$$

Therefore,

$$
\begin{aligned}
\left\|\varrho_{b c b}\left(\sigma_{\alpha}, \sigma_{\beta}\right)\right\| & \leq \sum_{i=1}^{\beta-\alpha} 2^{i+\alpha-1}\left\|\varrho_{b c b}\left(\sigma_{0}, \sigma_{1}\right)\right\|=\sum_{t=\alpha}^{\beta-1} 2^{t}\left\|\varrho_{b c b}\left(\sigma_{0}, \sigma_{1}\right)\right\| \\
& \leq \sum_{i=\alpha}^{\infty} 2^{t}\left\|\varrho_{b c b}\left(\sigma_{0}, \sigma_{1}\right)\right\| \\
& =\frac{2^{\alpha}}{1-\imath}\left\|\varrho_{b c b}\left(\sigma_{0}, \sigma_{1}\right)\right\| .
\end{aligned}
$$

Hence, we have

$$
\left\|\varrho_{b c b}\left(\sigma_{\alpha}, \sigma_{\beta}\right)\right\| \leq \frac{2^{\alpha}}{1-2}\left\|\varrho_{b c b}\left(\sigma_{0}, \sigma_{1}\right)\right\| \rightarrow 0 \quad \text { as } \quad \alpha \rightarrow \infty .
$$

Hence, $\left\{\sigma_{\alpha}\right\}$ is a Cauchy sequence in $\mathfrak{W}$. In all the above discussed cases, we get that the sequence $\left\{\sigma_{\alpha}\right\}_{\alpha \in \mathbb{N}}$ is a Cauchy sequence. Since $\mathfrak{W}$ is complete, there exists $\sigma^{*} \in \mathfrak{W}$ such that $\sigma_{\alpha} \rightarrow \sigma^{*}$ as $\alpha \rightarrow \infty$ and

$$
\varrho_{b c b}\left(\sigma^{*}, \sigma^{*}\right)=\lim _{\alpha \rightarrow \infty} \varrho_{b c b}\left(\sigma^{*}, \sigma_{\alpha}\right)=\lim _{\alpha \rightarrow \infty} \varrho_{b c b}\left(\sigma_{\alpha}, \sigma_{\alpha}\right)=0 .
$$

By the continuity of $\Gamma$, it follows that $\sigma_{2 \alpha+1}=\Gamma \sigma_{2 \alpha} \rightarrow \Gamma \sigma^{*}$ as $\alpha \rightarrow \infty$.

$$
\text { i.e., } \varrho_{b c b}\left(\Gamma \sigma^{*}, \Gamma \sigma^{*}\right)=\lim _{\alpha \rightarrow \infty} \varrho_{b c b}\left(\Gamma \sigma^{*}, \Gamma \sigma_{2 \alpha}\right)=\lim _{\alpha \rightarrow \infty} \varrho_{b c b}\left(\Gamma \sigma_{2 \alpha}, \Gamma \sigma_{2 \alpha}\right) \text {. }
$$


However,

$$
\varrho_{b c b}\left(\Gamma \sigma^{*}, \Gamma \sigma^{*}\right)=\lim _{\alpha \rightarrow \infty} \varrho_{b c b}\left(\Gamma \sigma_{2 \alpha}, \Gamma \sigma_{2 \alpha}\right)=\lim _{\alpha \rightarrow \infty} \varrho_{b c b}\left(\sigma_{2 \alpha+1}, \sigma_{2 \alpha+1}\right)=0 .
$$

Next, we have to prove that $\sigma^{*}$ is a fixed point of $\Gamma$.

$$
\varrho_{b c b}\left(\Gamma \sigma^{*}, \sigma^{*}\right) \preceq_{i_{2}} \varrho_{b c b}\left(\Gamma \sigma^{*}, \Gamma \sigma_{2 \alpha}\right)+\varrho_{b c b}\left(\Gamma \sigma_{2 \alpha}, \sigma^{*}\right)-\varrho_{b c b}\left(\Gamma \sigma_{2 \alpha}, \Gamma \sigma_{2 \alpha}\right) .
$$

As $\alpha \rightarrow \infty$, we obtain $\left\|\varrho_{b c b}\left(\Gamma \sigma^{*}, \sigma^{*}\right)\right\| \leq 0$. Thus, $\wp_{b c b}\left(\Gamma \sigma^{*}, \sigma^{*}\right)=0$. Hence, $\varrho_{b c b}\left(\sigma^{*}, \sigma^{*}\right)=\varrho_{b c b}\left(\sigma^{*}, \Gamma \sigma^{*}\right)=\varrho_{b c b}\left(\Gamma \sigma^{*}, \Gamma \sigma^{*}\right)=0$ and $\Gamma \sigma^{*}=\sigma^{*}$. In the same way, we have $\sigma^{*} \in \mathfrak{W}$ such that $\sigma_{\alpha} \rightarrow \sigma^{*}$ as $\alpha \rightarrow \infty$ and

$$
\varrho_{b c b}\left(\sigma^{*}, \sigma^{*}\right)=\lim _{\alpha \rightarrow \infty} \varrho_{b c b}\left(\sigma^{*}, \sigma_{\alpha}\right)=\lim _{\alpha \rightarrow \infty} \varrho_{b c b}\left(\sigma_{\alpha}, \sigma_{\alpha}\right)=0 .
$$

By the continuity of $\Gamma$, it follows $\sigma_{2 \alpha+2}=\Lambda \sigma_{2 \alpha+1} \rightarrow \Lambda \sigma^{*}$ as $\alpha \rightarrow \infty$.

$$
\text { i.e., } \varrho_{b c b}\left(\Lambda \sigma^{*}, \Lambda \sigma^{*}\right)=\lim _{\alpha \rightarrow \infty} \varrho_{b c b}\left(\Lambda \sigma^{*}, \Lambda \sigma_{2 \alpha+1}\right)=\lim _{\alpha \rightarrow \infty} \varrho_{b c b}\left(\Lambda \sigma_{2 \alpha+1}, \Lambda \sigma_{2 \alpha+1}\right) \text {. }
$$

However,

$$
\varrho_{b c b}\left(\Lambda \sigma^{*}, \Lambda \sigma^{*}\right)=\lim _{\alpha \rightarrow \infty} \varrho_{b c b}\left(\Lambda \sigma_{2 \alpha+1}, \Lambda \sigma_{2 \alpha+1}\right)=\lim _{\alpha \rightarrow \infty} \varrho_{b c b}\left(\sigma_{2 \alpha+2}, \sigma_{2 \alpha+2}\right)=0 .
$$

Next, we have to prove that $\sigma^{*}$ is a fixed point of $\Lambda$.

$$
\varrho_{b c b}\left(\Lambda \sigma^{*}, \sigma^{*}\right) \preceq_{i_{2}} \varrho_{b c b}\left(\Lambda \sigma^{*}, \Lambda \sigma_{2 \alpha+1}\right)+\varrho_{b c b}\left(\Lambda \sigma_{2 \alpha+1}, \sigma^{*}\right)-\varrho_{b c b}\left(\Lambda \sigma_{2 \alpha+1}, \Gamma \sigma_{2 \alpha+1}\right) .
$$

As $\alpha \rightarrow \infty$, we obtain $\left\|\varrho_{b c b}\left(\Lambda \sigma^{*}, \sigma^{*}\right)\right\| \leq 0$. Thus, $\wp_{b c b}\left(\Lambda \sigma^{*}, \sigma^{*}\right)=0$. Hence, $\varrho_{b c b}\left(\sigma^{*}, \sigma^{*}\right)=\varrho_{b c b}\left(\sigma^{*}, \Lambda \sigma^{*}\right)=\varrho_{b c b}\left(\Lambda \sigma^{*}, \Lambda \sigma^{*}\right)=0$ and $\Lambda \sigma^{*}=\sigma^{*}$. Therefore, $\sigma^{*}$ is a common fixed point of the pair $(\Gamma, \Lambda)$.

To prove uniqueness, let us consider $\psi^{*} \in \mathfrak{W}$ as another common fixed point for the pair $(\Gamma, \Lambda)$. Then,

$$
\begin{aligned}
& \varrho_{b c b}\left(\sigma^{*}, \psi^{*}\right)= \varrho_{b c b}\left(\Gamma \sigma^{*}, \Lambda \psi^{*}\right) \\
& \preceq_{i_{2}} \curlywedge \max \left\{\varrho_{b c b}\left(\sigma^{*}, \psi^{*}\right), \varrho_{b c b}\left(\sigma^{*}, \Gamma \sigma^{*}\right), \varrho_{b c b}\left(\psi^{*}, \Lambda \psi^{*}\right),\right. \\
&\left.\frac{1}{2}\left(\varrho_{b c b}\left(\sigma^{*}, \Lambda \psi^{*}\right)+\varrho_{b c b}\left(\psi^{*}, \Gamma \sigma^{*}\right)\right)\right\} \\
& \preceq_{i_{2}} \curlywedge \max \left\{\varrho_{b c b}\left(\sigma^{*}, \psi^{*}\right), \varrho_{b c b}\left(\sigma^{*}, \sigma^{*}\right), \varrho_{b c b}\left(\psi^{*}, \psi^{*}\right),\right. \\
&\left.\frac{1}{2}\left(\varrho_{b c b}\left(\sigma^{*}, \psi^{*}\right)+\varrho_{b c b}\left(\psi^{*}, \sigma^{*}\right)\right)\right\} \\
& \preceq_{i_{2}} \curlywedge \varrho_{b c b}\left(\sigma^{*}, \psi^{*}\right) .
\end{aligned}
$$

This implies that $\sigma^{*}=\psi^{*}$.

In the absence of the continuity condition for the mappings $\Gamma$ and $\Lambda$, we get the the following theorem.

Theorem 4. Let $\left(\mathfrak{W}, \varrho_{b c b}\right)$ be a complete bicomplex partial metric space and $\Gamma, \Lambda: \mathfrak{W} \rightarrow \mathfrak{W}$ be two mappings such that

$$
\begin{aligned}
\varrho_{b c b}(\Gamma \sigma, \Lambda \psi) \preceq & {i_{2}}_{2} \curlywedge \max \left\{\varrho_{b c b}(\sigma, \psi), \varrho_{b c b}(\sigma, \Gamma \sigma), \varrho_{b c b}(\psi, \Lambda \psi),\right. \\
& \left.\frac{1}{2}\left(\varrho_{b c b}(\sigma, \Lambda \psi)+\varrho_{b c b}(\psi, \Gamma \sigma)\right)\right\},
\end{aligned}
$$

for all $\sigma, \psi \in \mathfrak{W}$, where $0 \leq \curlywedge<1$. Then, the pair $(\Gamma, \Lambda)$ has a unique common fixed point and $\varrho_{b c b}\left(\sigma^{*}, \sigma^{*}\right)=0$. 
Proof. Following from Theorem 3 , we get that the sequence $\left\{\sigma_{\alpha}\right\}$ is a Cauchy sequence. Since $\mathfrak{W}$ is complete, there exists $\sigma^{*} \in \mathfrak{W}$ such that $\sigma_{\alpha} \rightarrow \sigma^{*}$ as $\alpha \rightarrow \infty$.

Since $\Gamma$ and $\Lambda$ are not continuous, we have $\varrho_{b c b}\left(\sigma^{*}, \Gamma \sigma^{*}\right)=\vartheta>0$.

Then, we estimate

$$
\begin{aligned}
& \vartheta=\varrho_{b c b}\left(\sigma^{*}, \Gamma \sigma^{*}\right) \\
& \preceq_{i_{2}} \varrho_{b c b}\left(\sigma^{*}, \sigma_{2 \alpha+2}\right)+\varrho_{b c b}\left(\sigma_{2 \alpha+2}, \Gamma \sigma^{*}\right)-\varrho_{b c b}\left(\sigma_{2 \alpha+2}, \sigma_{2 \alpha+2}\right) \\
& \preceq_{i_{2}} \varrho_{b c b}\left(\sigma^{*}, \sigma_{2 \alpha+2}\right)+\varrho_{b c b}\left(\sigma_{2 \alpha+2}, \Gamma \sigma^{*}\right) \\
& \preceq_{i_{2}} \varrho_{b c b}\left(\sigma^{*}, \sigma_{2 \alpha+2}\right)+\varrho_{b c b}\left(\Lambda \sigma_{2 \alpha+1}, \Gamma \sigma^{*}\right) \\
& \preceq_{i_{2}} \varrho_{b c b}\left(\sigma^{*}, \sigma_{2 \alpha+2}\right)+\curlywedge \max \left\{\varrho_{b c b}\left(\sigma_{2 \alpha+1}, \sigma^{*}\right), \varrho_{b c b}\left(\sigma_{2 \alpha+1}, \Lambda \sigma_{2 \alpha+1}\right), \varrho_{b c b}\left(\sigma^{*}, \Gamma \sigma^{*}\right),\right. \\
& \left.\frac{1}{2}\left(\varrho_{b c b}\left(\sigma_{2 \alpha+1}, \Gamma \sigma^{*}\right)+\varrho_{b c b}\left(\sigma^{*}, \Lambda \sigma_{2 \alpha+1}\right)\right)\right\} \\
& \preceq_{i_{2}} \varrho_{b c b}\left(\sigma^{*}, \sigma_{2 \alpha+2}\right)+\curlywedge \max \left\{\varrho_{b c b}\left(\sigma_{2 \alpha+1}, \sigma^{*}\right), \varrho_{b c b}\left(\sigma_{2 \alpha+1}, \sigma_{2 \alpha+2}\right), \varrho_{b c b}\left(\sigma^{*}, \Gamma \sigma^{*}\right)\right. \text {, } \\
& \left.\frac{1}{2}\left(\varrho_{b c b}\left(\sigma_{2 \alpha+1}, \Gamma \sigma^{*}\right)+\varrho_{b c b}\left(\sigma^{*}, \sigma_{2 \alpha+2}\right)\right)\right\} \\
& \preceq_{i_{2}} \varrho_{b c b}\left(\sigma^{*}, \sigma_{2 \alpha+2}\right)+\curlywedge \varrho_{b c b}\left(\sigma^{*}, \Gamma \sigma^{*}\right) \\
& \preceq_{i_{2}} \varrho_{b c b}\left(\sigma^{*}, \sigma_{2 \alpha+2}\right)+\curlywedge \vartheta \text {. }
\end{aligned}
$$

This yields

$$
\|\vartheta\| \leq\left\|\varrho_{b c b}\left(\sigma^{*}, \sigma_{2 \alpha+2}\right)\right\|+\curlywedge\|\vartheta\| .
$$

Hence, $\curlywedge \geq 1$, which is a contradiction. Then, $\sigma^{*}=\Gamma \sigma^{*}$. In the same way, we obtain $\sigma^{*}=\Lambda \sigma^{*}$. Hence, $\sigma^{*}$ is a common fixed point for the pair $(\Gamma, \Lambda)$ and $\varrho_{b c b}\left(\sigma^{*}, \sigma^{*}\right)=$ $\varrho_{b c b}\left(\sigma^{*}, \Lambda \sigma^{*}\right)=\varrho_{b c b}\left(\Lambda \sigma^{*}, \Lambda \sigma^{*}\right)=0$. For uniqueness of the common fixed point, $\sigma^{*}$ follows from Theorem 3.

For $\Gamma=\Lambda$, we get the following fixed points results on bicomplex partial metric space.

Theorem 5. Let $\left(\mathfrak{W}, \varrho_{b c b}\right)$ be a complete bicomplex partial metric space and $\Gamma: \mathfrak{W} \rightarrow \mathfrak{W}$ be a continuous mapping such that

$$
\begin{gathered}
\varrho_{b c b}(\Gamma \sigma, \Gamma \psi) \preceq i_{i_{2}} \curlywedge \max \left\{\varrho_{b c b}(\sigma, \psi), \varrho_{b c b}(\sigma, \Gamma \sigma), \varrho_{b c b}(\psi, \Gamma \psi),\right. \\
\\
\left.\frac{1}{2}\left(\varrho_{b c b}(\sigma, \Gamma \psi)+\varrho_{b c b}(\psi, \Gamma \sigma)\right)\right\},
\end{gathered}
$$

for all $\sigma, \psi \in \mathfrak{W}$, where $0 \leq \curlywedge<1$. Then, the pair $\Gamma$ has a unique fixed point and $\varrho_{b c b}\left(\sigma^{*}, \sigma^{*}\right)=0$.

Remark 1. Similarly, we get a fixed point result in the absence of continuity condition for the mapping $\Gamma$.

Corollary 1. Let $\left(\mathfrak{W}, \varrho_{b c b}\right)$ be a complete bicomplex partial metric space and $\Lambda: \mathfrak{W} \rightarrow \mathfrak{W}$ be a continuous mapping such that

$$
\begin{aligned}
\varrho_{b c b}\left(\Lambda^{\alpha} \sigma, \Lambda^{\alpha} \psi\right) \preceq & i_{2} \curlywedge \max \left\{\varrho_{b c b}(\sigma, \psi), \varrho_{b c b}\left(\sigma, \Lambda^{\alpha} \sigma\right), \varrho_{b c b}\left(\psi, \Lambda^{\alpha} \psi\right),\right. \\
& \left.\frac{1}{2}\left(\varrho_{b c b}\left(\sigma, \Lambda^{\alpha} \psi\right)+\varrho_{b c b}\left(\psi, \Lambda^{\alpha} \sigma\right)\right)\right\},
\end{aligned}
$$

for all $\sigma, \psi \in \mathfrak{W}$, where $0 \leq \curlywedge<1, \alpha \in \mathbb{N}$. Then, $\Lambda$ has a unique fixed point and $\varrho_{b c b}\left(\sigma^{*}, \sigma^{*}\right)=0$. 
Proof. By Theorem 3, we get $\sigma^{*} \in \mathfrak{W}$ such that $\Lambda^{\alpha} \sigma^{*}=\sigma^{*}$ and $\varrho_{b c b}\left(\sigma^{*}, \sigma^{*}\right)=0$. Then, we get

$$
\begin{aligned}
& \varrho_{b c b}\left(\Lambda \sigma^{*}, \sigma^{*}\right)= \varrho_{b c b}\left(\Lambda \Psi^{\alpha} \sigma^{*}, \Lambda^{n} \sigma^{*}\right)=\varrho_{b c b}\left(\Lambda^{\alpha} \Lambda \sigma^{*}, \Lambda^{\alpha} \sigma^{*}\right) \\
& \preceq_{i_{2}} \curlywedge \max \left\{\varrho_{b c b}\left(\Lambda \sigma^{*}, \sigma^{*}\right), \varrho_{b c b}\left(\Lambda \sigma^{*}, \Lambda^{\alpha} \Lambda \sigma^{*}\right), \varrho_{b c b}\left(\sigma^{*}, \Lambda^{\alpha} \sigma^{*}\right),\right. \\
&\left.\frac{1}{2}\left(\varrho_{b c b}\left(\Lambda \sigma^{*}, \Lambda^{\alpha} \sigma^{*}\right)+\varrho_{b c b}\left(\sigma^{*}, \Lambda^{\alpha} \Lambda \sigma^{*}\right)\right)\right\} \\
& \preceq_{i_{2}} \curlywedge \max \left\{\varrho_{b c b}\left(\Lambda \sigma^{*}, \sigma^{*}\right), \varrho_{b c b}\left(\Lambda \sigma^{*}, \Lambda \sigma^{*}\right), \varrho_{b c b}\left(\sigma^{*}, \sigma^{*}\right),\right. \\
&\left.\frac{1}{2}\left(\varrho_{b c b}\left(\Lambda \sigma^{*}, \sigma^{*}\right)+\varrho_{b c b}\left(\sigma^{*}, \Lambda \sigma^{*}\right)\right)\right\} \\
&= \curlywedge \varrho_{b c b}\left(\Lambda \sigma^{*}, \sigma^{*}\right) .
\end{aligned}
$$

Hence, $\Lambda^{\alpha} \sigma^{*}=\Lambda \sigma^{*}=\sigma^{*}$. Then, $\Lambda$ has a unique fixed point.

Remark 2. From the above Corollary 1, similarly, we get a fixed point result in the absence of continuity condition for the mapping $\Lambda$.

Next, we will present a new generalization of a common fixed point theorem on bicomplex partial metric space.

Theorem 6. Let $\left(\mathfrak{W}, \varrho_{b c b}\right)$ be a complete bicomplex partial metric space with non singular $1+$ $\varrho_{b c b}(\sigma, \psi)$ and $\left\|1+\varrho_{b c b}(\sigma, \psi)\right\| \neq 0$ and $\Gamma, \Lambda: \mathfrak{W} \rightarrow \mathfrak{W}$ be two continuous mappings such that

$$
\begin{aligned}
\varrho_{b c b}(\Gamma \sigma, \Lambda \psi) \preceq & i_{2} \curlywedge \max \left\{\varrho_{b c b}(\sigma, \psi), \frac{\varrho_{b c b}(\sigma, \Gamma \sigma) \varrho_{b c b}(\psi, \Lambda \psi)}{1+\varrho_{b c b}(\sigma, \psi)},\right. \\
& \left.\frac{\varrho_{b c b}(\sigma, \Gamma \sigma) \varrho_{b c b}(\Gamma \sigma, \Lambda \psi)}{1+\varrho_{b c b}(\sigma, \psi)}\right\},
\end{aligned}
$$

for all $\sigma, \psi \in \mathfrak{W}$, where $0 \leq \curlywedge<1$. Then, the pair $(\Gamma, \Lambda)$ has a unique common fixed point and $\varrho_{b c b}\left(\sigma^{*}, \sigma^{*}\right)=0$.

Proof. Let $\sigma_{0}$ be arbitrary point in $\mathfrak{W}$ and define a sequence $\left\{\sigma_{\alpha}\right\}$ as follows:

$$
\sigma_{2 \alpha+1}=\Gamma \sigma_{2 \alpha} \quad \text { and } \quad \sigma_{2 \alpha+2}=\Lambda \sigma_{2 \alpha+1}, \alpha=0,1,2, \ldots
$$

Then, by (12) and (13), we obtain

$$
\begin{aligned}
& \varrho_{b c b}\left(\sigma_{2 \alpha+1}, \sigma_{2 \alpha+2}\right)= \varrho_{b c b}\left(\Gamma \sigma_{2 \alpha}, \Lambda \sigma_{2 \alpha+1}\right) \\
& \preceq_{i_{2}} \curlywedge \max \left\{\varrho_{b c b}\left(\sigma_{2 \alpha}, \sigma_{2 \alpha+1}\right),\right. \\
& \frac{\varrho_{b c b}\left(\sigma_{2 \alpha}, \sigma_{2 \alpha+1}\right) \varrho_{b c b}\left(\Lambda \sigma_{2 \alpha+1}, \Gamma \sigma_{2 \alpha}\right)}{1+\varrho_{b c b}\left(\sigma_{2 \alpha}, \sigma_{2 \alpha+1}\right)}, \\
&\left.\frac{\varrho_{b c b}\left(\sigma_{2 \alpha}, \sigma_{2 \alpha+1}\right) \varrho_{b c b}\left(\Gamma \sigma_{2 \alpha}, \Lambda \sigma_{2 \alpha+1}\right)}{1+\varrho_{b c b}\left(\sigma_{2 \alpha}, \sigma_{2 \alpha+1}\right)}\right\} \\
& \preceq_{i_{2}} \curlywedge \max \left\{\varrho_{b c b}\left(\sigma_{2 \alpha}, \sigma_{2 \alpha+1}\right),\right. \\
& \frac{\varrho_{b c b}\left(\sigma_{2 \alpha}, \sigma_{2 \alpha+1}\right) \varrho_{b c b}\left(\sigma_{2 \alpha+1}, \sigma_{2 \alpha+2}\right)}{1+\varrho_{b c b}\left(\sigma_{2 \alpha}, \sigma_{2 \alpha+1}\right)}, \\
&\left.\frac{\varrho_{b c b}\left(\sigma_{2 \alpha}, \sigma_{2 \alpha+1}\right) \varrho_{b c b}\left(\sigma_{2 \alpha+1}, \sigma_{2 \alpha+2}\right)}{1+\varrho_{b c b}\left(\sigma_{2 \alpha}, \sigma_{2 \alpha+1}\right)}\right\} \\
& \preceq_{i_{2}} \curlywedge \max \left\{\varrho_{b c b}\left(\sigma_{2 \alpha}, \sigma_{2 \alpha+1}\right), \varrho_{b c b}\left(\sigma_{2 \alpha+1}, \sigma_{2 \alpha+2}\right)\right\} . \\
& \text { If } \max \left\{\varrho_{b c b}\left(\sigma_{2 \alpha}, \sigma_{2 \alpha+1}\right), \varrho_{b c b}\left(\sigma_{2 \alpha+1}, \sigma_{2 \alpha+2}\right)\right\}=\varrho_{b c b}\left(\sigma_{2 \alpha+1}, \sigma_{2 \alpha+2}\right), \text { then } \\
& \varrho_{b c b}\left(\sigma_{2 \alpha+1}, \sigma_{2 \alpha+2}\right) \preceq_{i_{2}} \curlywedge \varrho_{b c b}\left(\sigma_{2 \alpha+1}, \sigma_{2 \alpha+2}\right) .
\end{aligned}
$$


This shows that $\curlywedge \geq 1$, which is a contradiction. Therefore,

$$
\varrho_{b c b}\left(\sigma_{2 \alpha+1}, \sigma_{2 \alpha+2}\right) \preceq_{i_{2}} \curlywedge \varrho_{b c b}\left(\sigma_{2 \alpha}, \sigma_{2 \alpha+1}\right) .
$$

Similarly, we obtain

$$
\varrho_{b c b}\left(\sigma_{2 \alpha+2}, \sigma_{2 \alpha+3}\right) \preceq_{i_{2}} \curlywedge \varrho_{b c b}\left(\sigma_{2 \alpha+1}, \sigma_{2 \alpha+2}\right) .
$$

From (14) and (15), $\forall \alpha=0,1,2, \ldots$, we get

$$
\varrho_{b c b}\left(\sigma_{\alpha+1}, \sigma_{\alpha+2}\right) \preceq_{i_{2}} \curlywedge \varrho_{b c b}\left(\sigma_{\alpha}, \sigma_{\alpha+1}\right) \preceq_{i_{2}} \ldots \preceq_{i_{2}} \curlywedge^{\alpha+1} \varrho_{b c b}\left(\sigma_{0}, \sigma_{1}\right) .
$$

For $\beta, \alpha \in \mathbb{N}$, with $\beta>\alpha$, we have

$$
\begin{aligned}
& \varrho_{b c b}\left(\sigma_{\alpha}, \sigma_{\beta}\right) \preceq_{i_{2}} \varrho_{b c b}\left(\sigma_{\alpha}, \sigma_{\alpha+1}\right)+\varrho_{b c b}\left(\sigma_{\alpha+1}, \sigma_{\beta}\right)-\varrho_{b c b}\left(\sigma_{\alpha+1}, \sigma_{\alpha+1}\right) \\
& \preceq_{i_{2}} \varrho_{b c b}\left(\sigma_{\alpha}, \sigma_{\alpha+1}\right)+\varrho_{b c b}\left(\sigma_{\alpha+1}, \sigma_{\beta}\right) \\
& \preceq_{i_{2}} \varrho_{b c b}\left(\sigma_{\alpha}, \sigma_{\alpha+1}\right)+\varrho_{b c b}\left(\sigma_{\alpha+1}, \sigma_{\alpha+2}\right)+\varrho_{b c b}\left(\sigma_{\alpha+2}, \sigma_{\beta}\right) \\
&-\varrho_{b c b}\left(\sigma_{\alpha+2}, \sigma_{\alpha+2}\right) \\
& \preceq_{i_{2}} \varrho_{b c b}\left(\sigma_{\alpha}, \sigma_{\alpha+1}\right)+\varrho_{b c b}\left(\sigma_{\alpha+1}, \sigma_{\alpha+2}\right)+\varrho_{b c b}\left(\sigma_{\alpha+2}, \sigma_{\beta}\right) \\
& \preceq_{i_{2}} \varrho_{b c b}\left(\sigma_{\alpha}, \sigma_{\alpha+1}\right)+\varrho_{b c b}\left(\sigma_{\alpha+1}, \sigma_{\alpha+2}\right)+\varrho_{b c b}\left(\sigma_{\alpha+2}, \sigma_{\alpha+3}\right) \\
&+\ldots+\varrho_{b c b}\left(\sigma_{\beta-2}, \sigma_{\beta-1}\right)+s^{\beta-\alpha} \varrho_{b c b}\left(\sigma_{\beta-1}, \sigma_{\beta}\right) .
\end{aligned}
$$

By using (16), we get

$$
\begin{aligned}
& \varrho_{b c b}\left(\sigma_{\alpha}, \sigma_{\beta}\right) \preceq_{i_{2}} \curlywedge^{\alpha} \varrho_{b c b}\left(\sigma_{0}, \sigma_{1}\right)+\curlywedge^{\alpha+1} \varrho_{b c b}\left(\sigma_{0}, \sigma_{1}\right)+\curlywedge^{\alpha+2} \varrho_{b c b}\left(\sigma_{0}, \sigma_{1}\right) \\
&+\ldots+\curlywedge^{\beta-2} \varrho_{b c b}\left(\sigma_{0}, \sigma_{1}\right)+\curlywedge^{\beta-1} \varrho_{b c b}\left(\sigma_{0}, \sigma_{1}\right) \\
&= \sum_{i=1}^{\beta-\alpha} \curlywedge^{i+\alpha-1} \varrho_{b c b}\left(\sigma_{0}, \sigma_{1}\right) .
\end{aligned}
$$

Therefore,

$$
\begin{aligned}
\left\|\varrho_{b c b}\left(\sigma_{\alpha}, \sigma_{\beta}\right)\right\| & \leq \sum_{i=1}^{\beta-\alpha} \curlywedge^{i+\alpha-1}\left\|\varrho_{b c b}\left(\sigma_{0}, \sigma_{1}\right)\right\|=\sum_{i=1}^{\beta-\alpha} \curlywedge^{t}\left\|\varrho_{b c b}\left(\sigma_{0}, \sigma_{1}\right)\right\| \\
& \leq \sum_{i=\alpha}^{\infty} \curlywedge^{t}\left\|\varrho_{b c b}\left(\sigma_{0}, \sigma_{1}\right)\right\| \\
& =\frac{\curlywedge^{\alpha}}{1-\curlywedge}\left\|\varrho_{b c b}\left(\sigma_{0}, \sigma_{1}\right)\right\| .
\end{aligned}
$$

Hence, we have

$$
\left\|\varrho_{b c b}\left(\sigma_{\alpha}, \sigma_{\beta}\right)\right\| \leq \frac{\curlywedge^{\alpha}}{1-\curlywedge}\left\|\varrho_{b c b}\left(\sigma_{0}, \sigma_{1}\right)\right\| \rightarrow 0 \quad \text { as } \quad \alpha \rightarrow \infty .
$$

Hence, $\left\{\sigma_{\alpha}\right\}$ is a Cauchy sequence in $\mathfrak{W}$. Since $\mathfrak{W}$ is complete, then there exists $\sigma^{*} \in \mathfrak{W}$ such that $\sigma_{\alpha} \rightarrow \sigma^{*}$ as $\alpha \rightarrow \infty$ and

$$
\varrho_{b c b}\left(\sigma^{*}, \sigma^{*}\right)=\lim _{\alpha \rightarrow \infty} \varrho_{b c b}\left(\sigma^{*}, \sigma_{\alpha}\right)=\lim _{\alpha \rightarrow \infty} \varrho_{b c b}\left(\sigma_{\alpha}, \sigma_{\alpha}\right)=0 .
$$

$\Lambda$ being continuous yields

$$
\sigma^{*}=\lim _{\alpha \rightarrow \infty} \sigma_{2 \alpha+2}=\lim _{\alpha \rightarrow \infty} \Lambda \sigma_{2 \alpha+1}=\Lambda \lim _{\alpha \rightarrow \infty} \sigma_{2 \alpha+1}=\Lambda \sigma^{*} .
$$


Similarly, by the continuity of $\Gamma$, we get $\sigma^{*}=\Gamma \sigma^{*}$. Then, the pair $(\Gamma, \Lambda)$ has a common fixed point. To prove uniqueness, let us consider that $\psi^{*} \in \mathfrak{W}$ is another common fixed point for the pair $(\Gamma, \Lambda)$. Then,

$$
\begin{aligned}
& \varrho_{b c b}\left(\sigma^{*}, \psi^{*}\right)= \varrho_{b c b}\left(\Gamma \sigma^{*}, \Lambda \psi^{*}\right) \\
& \preceq_{i_{2}} \curlywedge \max \left\{\varrho_{b c b}\left(\sigma^{*}, \psi^{*}\right), \frac{\varrho_{b c b}\left(\sigma^{*}, \Gamma \sigma^{*}\right) \varrho_{b c b}\left(\psi^{*}, \Lambda \psi^{*}\right)}{1+\varrho_{b c b}\left(\sigma^{*}, \psi^{*}\right)},\right.\left.\frac{\varrho_{b c b}\left(\sigma^{*}, \Gamma \sigma^{*}\right) \varrho_{b c b}\left(\Lambda \psi^{*}, \Gamma \sigma^{*}\right)}{1+\varrho_{b c b}\left(\sigma^{*}, \psi^{*}\right)}\right\} \\
& \preceq_{i_{2}} \curlywedge \varrho_{b c b}\left(\sigma^{*}, \psi^{*}\right) .
\end{aligned}
$$

This implies that $\sigma^{*}=\psi^{*}$.

In the absence of the continuity condition for the mapping $\Gamma$ and $\Lambda$ in Theorem 6 , we obtain the following result.

Theorem 7. Let $\left(\mathfrak{W}, \varrho_{b c b}\right)$ be a complete bicomplex partial metric space with non singular $1+$ $\varrho_{b c b}(\sigma, \psi)$ and $\left\|1+\varrho_{b c b}(\sigma, \psi)\right\| \neq 0$ and $\Gamma, \Lambda: \mathfrak{W} \rightarrow \mathfrak{W}$ be two mappings such that

$$
\begin{aligned}
\varrho_{b c b}(\Gamma \sigma, \Lambda \psi) \preceq & i_{2} \curlywedge \max \left\{\varrho_{b c b}(\sigma, \psi), \frac{\varrho_{b c b}(\sigma, \Gamma \sigma) \varrho_{b c b}(\psi, \Lambda \psi)}{1+\varrho_{b c b}(\sigma, \psi)},\right. \\
& \left.\frac{\varrho_{b c b}(\sigma, \Gamma \sigma) \varrho_{b c b}(\Gamma \sigma, \Lambda \psi)}{1+\varrho_{b c b}(\sigma, \psi)}\right\},
\end{aligned}
$$

for all $\sigma, \psi \in \mathfrak{W}$, where $0 \leq \curlywedge<1$. Then, the pair $(\Gamma, \Lambda)$ has a unique common fixed point and $\varrho_{b c b}\left(\sigma^{*}, \sigma^{*}\right)=0$.

Proof. Following from Theorem 6, we get that the sequence $\left\{\sigma_{\alpha}\right\}$ is a Cauchy sequence. Since $\mathfrak{W}$ is complete, then there exists $\sigma^{*} \in \mathfrak{W}$ such that $\sigma_{\alpha} \rightarrow \sigma^{*}$ as $\alpha \rightarrow \infty$ and

$$
\varrho_{b c b}\left(\sigma^{*}, \sigma^{*}\right)=\lim _{\alpha \rightarrow \infty} \varrho_{b c b}\left(\sigma^{*}, \sigma_{\alpha}\right)=\lim _{\alpha \rightarrow \infty} \varrho_{b c b}\left(\sigma_{\alpha}, \sigma_{\alpha}\right)=0 .
$$

Since $\Gamma$ and $\Lambda$ are not continuous, we have $\varrho_{b c b}\left(\sigma^{*}, \Gamma \sigma^{*}\right)=\vartheta>0$. Then, we estimate

$$
\begin{aligned}
& \vartheta=\varrho_{b c b}\left(\sigma^{*}, \Gamma \sigma^{*}\right) \\
& \varliminf_{i_{2}} \varrho_{b c b}\left(\sigma^{*}, \sigma_{2 \alpha+2}\right)+\varrho_{b c b}\left(\sigma_{2 \alpha+2}, \Gamma \sigma^{*}\right)-\varrho_{b c b}\left(\sigma_{2 \alpha+2}, \sigma_{2 \alpha+2}\right) \\
& \varliminf_{i_{2}} \varrho_{b c b}\left(\sigma^{*}, \sigma_{2 \alpha+2}\right)+\varrho_{b c b}\left(\Gamma \sigma^{*}, \sigma_{2 \alpha+2}\right) \\
& \varliminf_{i_{2}} \varrho_{b c b}\left(\sigma^{*}, \sigma_{2 \alpha+2}\right)+\varrho_{b c b}\left(\Gamma \sigma^{*}, \Lambda \sigma_{2 \alpha+1}\right) \\
& \varliminf_{i_{2}} \varrho_{b c b}\left(\sigma^{*}, \sigma_{2 \alpha+2}\right)+\curlywedge \max \left\{\varrho_{b c b}\left(\sigma^{*}, \sigma_{2 \alpha+1}\right), \frac{\varrho_{b c b}\left(\sigma^{*}, \Gamma \sigma^{*}\right) \varrho_{b c b}\left(\sigma_{2 \alpha+1}, \Lambda \sigma_{2 \alpha+1}\right)}{1+\varrho_{b c b}\left(\sigma^{*}, \sigma_{2 \alpha+1}\right)},\right. \\
&\left.\frac{\varrho_{b c b}\left(\sigma^{*}, \Gamma \sigma_{*}\right) \varrho_{b c b}\left(\Gamma \sigma^{*}, \Lambda \sigma_{2 \alpha+1}\right)}{1+\varrho_{b c b}\left(\sigma^{*}, \sigma_{2 \alpha+1}\right)}\right\} \\
& \preceq_{i_{2}} \varrho_{b c b}\left(\sigma^{*}, \sigma_{2 \alpha+2}\right)+\curlywedge \max \left\{\varrho_{b c b}\left(\sigma^{*}, \sigma_{2 \alpha+1}\right), \frac{\varrho_{b c b}\left(\sigma^{*}, \Gamma \sigma^{*}\right) \varrho_{b c b}\left(\sigma_{2 \alpha+1}, \sigma_{2 \alpha+2}\right)}{1+\varrho_{b c b}\left(\sigma^{*}, \sigma_{2 \alpha+1}\right)}\right\}, \\
&\left.\frac{\varrho_{b c b}\left(\sigma^{*}, \Gamma \sigma_{*}\right) \varrho_{b c b}\left(\Gamma \sigma^{*}, \sigma_{2 \alpha+2}\right)}{1+\varrho_{b c b}\left(\sigma^{*}, \sigma_{2 \alpha+1}\right)}\right\} \\
& \preceq_{i_{2}} \varrho_{b c b}\left(\sigma^{*}, \sigma_{2 \alpha+2}\right)+\curlywedge \varrho_{b c b}\left(\sigma^{*}, \Gamma \sigma^{*}\right)^{2} \\
& \preceq_{i_{2}} \varrho_{b c b}\left(\sigma^{*}, \sigma_{2 \alpha+2}\right)+\curlywedge \vartheta^{2} .
\end{aligned}
$$


This yields

$$
\|\vartheta\| \leq\left\|\varrho_{b c b}\left(\sigma^{*}, \sigma_{2 \alpha+2}\right)\right\|+\curlywedge\|\vartheta\|^{2}
$$

Hence, $\curlywedge \geq 1$, which is a contradiction. Then, $\sigma^{*}=\Gamma \sigma^{*}$. In the same way, we obtain $\sigma^{*}=\Lambda \sigma^{*}$. Hence, $\sigma^{*}$ is a common fixed point for the pair $(\Gamma, \Lambda)$. The uniqueness of the common fixed point $\sigma^{*}$ follows from Theorem 6.

For $\Gamma=\Lambda$, we get the following fixed points results on bicomplex partial metric space.

Theorem 8. Let $\left(\mathfrak{W}, \varrho_{b c b}\right)$ be a complete bicomplex partial metric space with non singular $1+$ $\varrho_{b c b}(\sigma, \psi)$ and $\left\|1+\varrho_{b c b}(\sigma, \psi)\right\| \neq 0$ and $\Gamma: \mathfrak{W} \rightarrow \mathfrak{W}$ be a continuous mapping such that

$$
\begin{aligned}
\varrho_{b c b}(\Gamma \sigma, \Gamma \psi) \preceq & \curlywedge \max \left\{\varrho_{b c b}(\sigma, \psi), \frac{\varrho_{b c b}(\sigma, \Gamma \sigma) \varrho_{b c b}(\psi, \Gamma \psi)}{1+\varrho_{b c b}(\sigma, \psi)},\right. \\
& \left.\frac{\varrho_{b c b}(\sigma, \Gamma \sigma) \varrho_{b c b}(\Gamma \sigma, \Gamma \psi)}{1+\varrho_{b c b}(\sigma, \psi)}\right\},
\end{aligned}
$$

for all $\sigma, \psi \in \mathfrak{W}$, where $0 \leq \curlywedge<1$. Then, $\Gamma$ has a unique fixed point and $\varrho_{b c b}\left(\sigma^{*}, \sigma^{*}\right)=0$.

Remark 3. Similarly, in the absence of the continuity condition, we can get a fixed point result on $\Gamma$.

Corollary 2. Let $\left(\mathfrak{W}, \varrho_{b c b}\right)$ be a complete bicomplex partial metric space with non singular $1+$ $\varrho_{b c b}(\sigma, \psi)$ and $\left\|1+\varrho_{b c b}(\sigma, \psi)\right\| \neq 0$ and $\Gamma: \mathfrak{W} \rightarrow \mathfrak{W}$ be a continuous mapping such that

$$
\begin{aligned}
\varrho_{b c b}\left(\Gamma^{\alpha} \sigma, \Gamma^{\alpha} \psi\right) \preceq & i_{2} \curlywedge \max \left\{\varrho_{b c b}(\sigma, \psi), \frac{\varrho_{b c b}\left(\sigma, \Gamma^{\alpha} \sigma\right) \varrho_{b c b}\left(\psi, \Gamma^{\alpha} \psi\right)}{1+\varrho_{b c b}(\sigma, \psi)},\right. \\
& \left.\frac{\varrho_{b c b}\left(\sigma, \Gamma^{\alpha} \sigma\right) \varrho_{b c b}\left(\Gamma^{\alpha} \sigma, \Gamma \psi\right)}{1+\varrho_{b c b}(\sigma, \psi)}\right\},
\end{aligned}
$$

for all $\sigma, \psi \in \mathfrak{W}$, where $0 \leq \curlywedge<1$. Then, $\Gamma$ has a unique fixed point and $\varrho_{b c b}\left(\sigma^{*}, \sigma^{*}\right)=0$.

Proof. By Theorem 6, we get $\sigma^{*} \in \mathfrak{W}$ such that $\Gamma^{\alpha} \sigma^{*}=\sigma^{*}$ and $\varrho_{b c b}\left(\sigma^{*}, \sigma^{*}\right)=0$. Then, we get

$$
\begin{aligned}
& \varrho_{b c b}\left(\Gamma \sigma^{*}, \sigma^{*}\right)= \varrho_{b c b}\left(\Gamma \Gamma^{\alpha} \sigma^{*}, \Gamma^{\alpha} \sigma^{*}\right)=\varrho_{b c b}\left(\Gamma^{\alpha} \Gamma \sigma^{*}, \Gamma^{\alpha} \sigma^{*}\right) \\
& \preceq_{i_{2}} \curlywedge \max \left\{\varrho_{b c b}\left(\Gamma \sigma^{*}, \sigma^{*}\right), \frac{\varrho_{b c b}\left(\Gamma \sigma^{*}, \Gamma^{\alpha} \Gamma \sigma^{*}\right) \varrho_{b c b}\left(\sigma^{*}, \Gamma^{\alpha} \sigma^{*}\right)}{1+\varrho_{b c b}\left(\Gamma \sigma^{*}, \sigma^{*}\right)},\right. \\
&\left.\frac{\varrho_{b c b}\left(\Gamma \sigma^{*}, \Gamma^{\alpha} \Gamma \sigma^{*}\right) \varrho_{b c b}\left(\Gamma^{\alpha} \Gamma \sigma^{*}, \Gamma^{\alpha} \sigma^{*}\right)}{1+\varrho_{b c b}\left(\Gamma \sigma^{*}, \sigma^{*}\right)}\right\} \\
& \preceq_{i_{2}} \curlywedge \max \left\{\varrho_{b c b}\left(\Gamma \sigma^{*}, \sigma^{*}\right), \frac{\varrho_{b c b}\left(\Gamma \sigma^{*}, \Gamma \Gamma^{\alpha} \sigma^{*}\right) \varrho_{b c b}\left(\sigma^{*}, \Gamma^{\alpha} \sigma^{*}\right)}{1+\varrho_{b c b}\left(\Gamma \sigma^{*}, \sigma^{*}\right)},\right. \\
& \\
&\left.\frac{\varrho_{b c b}\left(\Gamma \sigma^{*}, \Gamma \Gamma^{\alpha} \sigma^{*}\right) \varrho_{b c b}\left(\Gamma \Gamma^{\alpha} \sigma^{*}, \Gamma^{\alpha} \sigma^{*}\right)}{1+\varrho_{b c b}\left(\Gamma \sigma^{*}, \sigma^{*}\right)}\right\} \\
&= \curlywedge \varrho_{b c b}\left(\Gamma \sigma^{*}, \sigma^{*}\right) .
\end{aligned}
$$

Hence, $\Gamma^{\alpha} \sigma^{*}=\Gamma \sigma^{*}=\sigma^{*}$. Then, $\Gamma$ has a unique fixed point.

Remark 4. From the above Corollary 2, similarly, we get a fixed point result in the absence of continuity condition for the mapping $\Gamma$.

Example 2. Let $\mathfrak{W}=\{1,2,3,4\}$ be endowed with the order $\sigma \preceq_{i_{2}} \psi$ if and only if $\sigma \leq \psi$. Then, $\preceq_{i_{2}}$ is a partial order in $\mathfrak{W}$. Define the bicomplex partial metric space $\varrho_{b c b}: \mathfrak{W} \times \mathfrak{W} \rightarrow \mathbb{C}_{2}^{+}$ as follows: 


\begin{tabular}{ll}
\hline$(\sigma, \psi)$ & $\varrho_{b c b}(\sigma, \psi)$ \\
\hline$(1,1),(2,2)$ & 0 \\
\hline$(1,2),(2,1),(1,3),(3,1),(2,3),(3,2),(3,3)$ & $e^{i_{2} x}$ \\
\hline$(1,4),(4,1),(2,4),(4,2),(3,4),(4,3),(4,4)$ & $3 e^{i_{2} x}$ \\
\hline
\end{tabular}

Obviously, $\left(\mathfrak{W}, \varrho_{b c b}\right)$ is a complete bicomplex partial metric space for $x \in\left[0, \frac{\pi}{2}\right]$. Define $\Gamma, \Lambda: \mathfrak{W} \rightarrow \mathfrak{W}$ by $\Gamma \sigma=1$,

$$
\Lambda(\sigma)= \begin{cases}1 & \text { if } \sigma \in\{1,2,3\} \\ 2 & \text { if } \sigma=4\end{cases}
$$

Clearly, $\Gamma$ and $\Lambda$ are continuous functions. Now, for $\curlywedge=\frac{1}{3}$, we consider the following cases:

(A) If $\sigma=1$ and $\psi \in G-\{4\}$, then $\Gamma(\sigma)=\Lambda(\psi)=1$ and the conditions of Theorem 3 are satisfied.

(B) If $\sigma=1, \psi=4$, then $\Gamma \sigma=1, \Lambda \psi=2$,

$$
\begin{aligned}
& \varrho_{b c b}(\Gamma \sigma, \Lambda \psi)=e^{i_{2} x} \preceq_{i_{2}} 3 \curlywedge e^{i_{2} x} \\
&=\curlywedge \max \left\{3 e^{i_{2} x}, 0,3 e^{i_{2} x},\right. \\
&\left.\frac{1}{2}\left(e^{i_{2} x}+3 e^{i_{2} x}\right)\right\} \\
&= \curlywedge \max \left\{\varrho_{b c b}(\sigma, \psi), \varrho_{b c b}(\sigma, \Gamma \sigma), \varrho_{b c b}(\psi, \Lambda \psi),\right. \\
&\left.\frac{1}{2}\left(\varrho_{b c b}(\sigma, \Lambda \psi)+\varrho_{b c b}(\psi, \Gamma \sigma)\right)\right\},
\end{aligned}
$$

(C) If $\sigma=2, \psi=4$, then $\Gamma \sigma=1, \Lambda \psi=2$,

$$
\begin{aligned}
& \varrho_{b c b}(\Gamma \sigma, \Lambda \psi)=e^{i_{2} x} \preceq_{i_{2}} 3 \curlywedge e^{i_{2} x} \\
&= \curlywedge \max \left\{3 e^{i_{2} x}, e^{i_{2} x}, 3 e^{i_{2} x},\right. \\
&\left.\frac{1}{2}\left(0+3 e^{i_{2} x}\right)\right\} \\
&= \curlywedge \max \left\{\varrho_{b c b}(\sigma, \psi), \varrho_{b c b}(\sigma, \Gamma \sigma), \varrho_{b c b}(\psi, \Lambda \psi),\right. \\
&\left.\frac{1}{2}\left(\varrho_{b c b}(\sigma, \Lambda \psi)+\varrho_{b c b}(\psi, \Gamma \sigma)\right)\right\},
\end{aligned}
$$

(D) If $\sigma=3, \psi=4$, then $\Gamma \sigma=1, \Lambda \psi=2$,

$$
\begin{aligned}
\varrho_{b c b}(\Gamma \sigma, \Lambda \psi)=e^{i_{2} x} \preceq & i_{2} 3 \curlywedge e^{i_{2} x} \\
= & \curlywedge \max \left\{3 e^{i_{2} x}, e^{i_{2} x}, 3 e^{i_{2} x},\right. \\
& \left.\frac{1}{2}\left(e^{i_{2} x}+3 e^{i_{2} x}\right)\right\} \\
= & \curlywedge \max \left\{\varrho_{b c b}(\sigma, \psi), \varrho_{b c b}(\sigma, \Gamma \sigma), \varrho_{b c b}(\psi, \Lambda \psi),\right. \\
& \left.\frac{1}{2}\left(\varrho_{b c b}(\sigma, \Lambda \psi)+\varrho_{b c b}(\psi, \Gamma \sigma)\right)\right\},
\end{aligned}
$$


(E) If $\sigma=4, \psi=4$, then $\Gamma \sigma=2, \Lambda \psi=2$,

$$
\begin{aligned}
& \varrho_{b c b}(\Gamma \sigma, \Lambda \psi)=e^{i_{2} x} \preceq_{2_{2}} 3 \curlywedge e^{i_{2} x} \\
&=\curlywedge \max \left\{3 e^{i_{2} x}, 3 e^{i_{2} x}, 3 e^{i_{2} x},\right. \\
&\left.\frac{1}{2}\left(3 e^{i_{2} x}+3 e^{i_{2} x}\right)\right\} \\
&= \curlywedge \max \left\{\varrho_{b c b}(\sigma, \psi), \varrho_{b c b}(\sigma, \Gamma \sigma), \varrho_{b c b}(\psi, \Lambda \psi),\right. \\
&\left.\frac{1}{2}\left(\varrho_{b c b}(\sigma, \Lambda \psi)+\varrho_{b c b}(\psi, \Gamma \sigma)\right)\right\},
\end{aligned}
$$

Moreover, for $\curlywedge=\frac{1}{3}$, with $\curlywedge<1$, the conditions of Theorem 3 are satisfied. Therefore, 1 is the unique common fixed point of $\Gamma$ and $\Lambda$.

\section{Application}

Let $\mathfrak{W}=C\left[\lambda_{1}, \lambda_{2}\right]$ be a set of all real continuous functions on $\left[\lambda_{1}, \lambda_{2}\right]$ equipped with metric $\varrho_{b c b}(\sigma, \psi)=\left(1+i_{2}\right)\left(\max _{\sqcup \in\left[\lambda_{1}, \lambda_{2}\right]}|\sigma(\sqcup)-\psi(\sqcup)|+2\right)$ for all $\sigma, \psi \in C\left[\lambda_{1}, \lambda_{2}\right]$, where |.| is the usual real modulus. Then, $\left(\mathfrak{W}, \varrho_{b c b}\right)$ is a complete bicomplex partial metric space. Now, we consider the system of nonlinear Fredholm integral equation

$$
\sigma(\sqcup)=\mathfrak{v}(\sqcup)+\frac{1}{\lambda_{2}-\lambda_{1}} \int_{\lambda_{1}}^{\lambda_{2}} \mathfrak{K}_{1}(\sqcup, \mathfrak{s}, \sigma(s)) d s
$$

and

$$
\sigma(\sqcup)=\mathfrak{v}(\sqcup)+\frac{1}{\lambda_{2}-\lambda_{1}} \int_{\lambda_{1}}^{\lambda_{2}} \mathfrak{K}_{2}(\sqcup, \mathfrak{s}, \sigma(s)) d s,
$$

where $\sqcup, \mathfrak{s} \in\left[\lambda_{1}, \lambda_{2}\right]$. Assume that $\mathfrak{K}_{1}, \mathfrak{K}_{2}:\left[\lambda_{1}, \lambda_{2}\right] \times\left[\lambda_{1}, \lambda_{2}\right] \times \mathfrak{W} \rightarrow \mathbb{R}$ and $\mathfrak{v}:$ $\left[\lambda_{1}, \lambda_{2}\right] \rightarrow \mathbb{R}$ are continuous, where $\mathfrak{v}(\sqcup)$ is a given function in $\mathfrak{W}$.

Theorem 9. Suppose that $(\mathfrak{W}, d)$ is a bicomplex partial metric space equipped with metric $\varrho_{b c b}(\sigma, \psi)=\left(1+i_{2}\right)\left(\max _{\sqcup \in\left[\lambda_{1}, \lambda_{2}\right]}|\sigma(\sqcup)-\psi(\sqcup)|+2\right)$ for all $\sigma, \psi \in \mathfrak{W}$ and $\Gamma, \Lambda: \mathfrak{W} \rightarrow \mathfrak{W}$ be a continuous operator on $\mathfrak{W}$ defined by

$$
\Gamma \sigma(\sqcup)=\mathfrak{v}(\sqcup)+\frac{1}{\lambda_{2}-\lambda_{1}} \int_{\lambda_{1}}^{\lambda_{2}} \mathfrak{K}_{1}(\sqcup, \mathfrak{s}, \sigma(s)) d s
$$

and

$$
\Lambda \sigma(\sqcup)=\mathfrak{v}(\sqcup)+\frac{1}{\lambda_{2}-\lambda_{1}} \int_{\lambda_{1}}^{\lambda_{2}} \mathfrak{K}_{2}(\sqcup, \mathfrak{s}, \sigma(s)) d s .
$$

If there exists $\iota>0$ such that, for all $\sigma, \psi \in \mathfrak{W}$ with $\sigma \neq \psi$ and $\mathfrak{s}, \sqcup \in\left[\lambda_{1}, \lambda_{2}\right]$ satisfying the following inequality:

$$
\begin{aligned}
\left|\mathfrak{K}_{1}(\sqcup, \mathfrak{s}, \sigma(s))-\mathfrak{K}_{2}(\sqcup, \mathfrak{s}, \psi(\mathfrak{s}))\right| \leq & \max \{|\sigma(s)-\psi(\mathfrak{s})|,|\sigma(s)-\Gamma \sigma(\mathfrak{s})|, \\
& |\psi(s)-\Lambda \psi(\mathfrak{s})|, \\
& \left.\frac{1}{2}(|\sigma(s)-\Lambda \psi(s)|+|\psi(s)-\Gamma \sigma(s)|)\right\},
\end{aligned}
$$

then the integral operators defined by (18) and (19) have a common unique solution. 
Proof. Consider,

$$
\begin{aligned}
\left(1+i_{2}\right)(|\Gamma \sigma(\sqcup)-\Lambda \psi(\sqcup)|+2)= & \frac{\left(1+i_{2}\right)}{\left|\lambda_{2}-\lambda_{1}\right|}\left(\mid \int_{\lambda_{1}}^{\lambda_{2}} \mathfrak{K}_{1}(\sqcup, \mathfrak{s}, \sigma(s)) d s\right. \\
& \left.-\int_{\lambda_{1}}^{\lambda_{2}} \mathfrak{K}_{2}(\sqcup, \mathfrak{s}, \psi(\mathfrak{s})) d s \mid+2\right) \\
\leq & \frac{\left(1+i_{2}\right)}{\left|\lambda_{2}-\lambda_{1}\right|}\left(\int_{\lambda_{1}}^{\lambda_{2}} \mid \mathfrak{K}_{1}(\sqcup, \mathfrak{s}, \sigma(s))\right. \\
& \left.-\mathfrak{K}_{2}(\sqcup, \mathfrak{s}, \psi(\mathfrak{s})) \mid d s+2\right) \\
\leq & \frac{\left(1+i_{2}\right) 2}{\left|\lambda_{2}-\lambda_{1}\right|}\left(\int_{\lambda_{1}}^{\lambda_{2}} \max \{|\sigma(s)-\psi(\mathfrak{s})|,\right. \\
& |\sigma(s)-\Gamma \sigma(\mathfrak{s})|,|\psi(s)-\Lambda \psi(\mathfrak{s})|, \\
& \left.\left.\frac{1}{2}(|\sigma(s)-\Lambda \psi(s)|+|\psi(s)-\Gamma \sigma(s)|)\right\}+2\right) \\
\leq & \frac{2}{\left|\lambda_{2}-\lambda_{1}\right|} \int_{\lambda_{1}}^{\lambda_{2}} \max \left\{\left(1+i_{2}\right)|\sigma(s)-\psi(\mathfrak{s})|+2,\right. \\
& \left(1+i_{2}\right)|\sigma(s)-\Gamma \sigma(\mathfrak{s})|+2, \\
& \left(1+i_{2}\right)|\psi(s)-\Lambda \psi(\mathfrak{s})|+2, \\
& \frac{1}{2}\left(\left(1+i_{2}\right)|\sigma(s)-\Lambda \psi(s)|+2\right. \\
& \left.\left.+\left(1+i_{2}\right)|\psi(s)-\Gamma \sigma(s)|+2\right)\right\} .
\end{aligned}
$$

Taking the maximum on both sides for all $\sqcup \in\left[\lambda_{1}, \lambda_{2}\right]$, we obtain

$$
\begin{aligned}
\left.\varrho_{b c b}(\Gamma \sigma, \Gamma \psi)\right)= & \left(1+i_{2}\right)\left(\max _{\sqcup \in\left[\lambda_{1}, \lambda_{2}\right]}|\Gamma \sigma(\sqcup)-\Lambda \psi(\sqcup)|+2\right) \\
\leq & \frac{2}{\left|\lambda_{2}-\lambda_{1}\right|} \max _{\sqcup \in\left[\lambda_{1}, \lambda_{2}\right]} \int_{\lambda_{1}}^{\lambda_{2}} \max \left\{\left(1+i_{2}\right)|\sigma(s)-\psi(\mathfrak{s})|+2,\right. \\
& \left(1+i_{2}\right)|\sigma(s)-\Gamma \sigma(\mathfrak{s})|+2,\left(1+i_{2}\right)|\psi(s)-\Lambda \psi(\mathfrak{s})|+2, \\
& \left.\frac{1}{2}\left(\left(1+i_{2}\right)|\sigma(s)-\Lambda \psi(s)|+2+\left(1+i_{2}\right)|\psi(s)-\Gamma \sigma(s)|+2\right)\right\} \\
\leq & \frac{2}{\left|\lambda_{2}-\lambda_{1}\right|} \max \left[\operatorname { m a x } _ { \nabla \in [ \lambda _ { 1 } , \lambda _ { 2 } ] } \left\{\left(1+i_{2}\right)|\sigma(\nabla)-\psi(\nabla)|+2,\right.\right. \\
& \left(1+i_{2}\right)|\sigma(\nabla)-\Gamma \psi(\nabla)|+2,\left(1+i_{2}\right)|\psi(\nabla)-\Gamma \psi(\nabla)|+2, \\
& \frac{1}{2}\left(\left(1+i_{2}\right)|\sigma(\nabla)-\Lambda \psi(\nabla)|+2\right. \\
& \left.\left.\left.+\left(1+i_{2}\right)|\psi(\nabla)-\Gamma \sigma(\nabla)|+2\right)\right\}\right] \int_{\lambda_{1}}^{\lambda_{2}} d s \\
= & \curlywedge \max \left\{\varrho_{b c b}(\sigma, \psi), \varrho_{b c b}(\sigma, \Gamma \sigma), \varrho_{b c b}(\psi, \Lambda \psi),\right. \\
& \left.\frac{1}{2}\left(\varrho_{b c b}(\sigma, \Lambda \psi)+\varrho_{b c b}(\psi, \Gamma \sigma)\right)\right\} .
\end{aligned}
$$

Hence, all the conditions of Theorem 3 are satisfied and so the integral operators $\Gamma$ and $\Lambda$ defined by (18) and (19) have a common unique solution.

\section{Conclusions}

In this paper, we proved some common fixed point theorems on bicomplex partial metric space. In addition, we find a common unique solution of a system of nonlinear 
Fredholm integral equations, and we support our theoretical results by an example that we explain.

Author Contributions: All authors contributed equally in writing this article. All authors read and approved the final manuscript.

Funding: This work was supported by the National Natural Science Foundation of P. R. China (Nos.11971493 and 12071491).

Institutional Review Board Statement: Not applicable.

Informed Consent Statement: Not applicable.

Data Availability Statement: Not applicable.

Conflicts of Interest: The authors declare no conflict of interest.

\section{References}

1. Segre, C. It Le Rappresentazioni Reali delle Forme Complesse a Gli Enti Iperalgebrici. Math. Ann. 1892, 40, 413-467. [CrossRef]

2. Dragoni, G.S. Sulle funzioni olomorfe di una variabile bicomplessa. Reale Accad. d'Italia. Mem. Classe Sci. Nat. Fis. Mat. 1934, 5, 597-665.

3. Spampinato, N. Estensione nel campo bicomplesso di due teoremi, del Levi-Civita e del Severi, per le funzioni olomorfe di due variablili bicomplesse I, II. Reale Accad. Naz. Lincei. 1935, 22, 38-43. 96-102.

4. Spampinato, N. Sulla rappresentazione delle funzioni do variabile bicomplessa totalmente derivabili. Ann. Mat. Pura Appl. 1936, 14, 305-325. [CrossRef]

5. Price, G.B. An Introduction to Multicomplex Spaces and Functions; Marcel Dekker: New York, NY, USA, 1991.

6. Colombo, F.; Sabadini, I.; Struppa, D.C.; Vajiac, A.; Vajiac, M. Singularities of functions of one and several bicomplex variables. Ark. Math 2010, 49, 277-294. [CrossRef]

7. Ragusa, M.A. Hölder regularity results for solutions of parabolic equations. In Variational Analysis and Applications; Nonconvex Optimization and Its Applications; Springer: New York, NY, USA, 2005 , Volume 79, pp. 921-934. ISBN 0-387-24209-0.

8. Yau, D. Deformation bicomplex of module algebras. Homol. Homotopy Appl. 2008, 10, 97-128. [CrossRef]

9. Kumar, R.; Singh, K.; Saini, H.; Kumar, S. Bicomplex weighted Hardy spaces and bicomplex C*-algebras. Adv. Appl. Clifford Algebras 2016, 26, 217-235. [CrossRef]

10. Luna-Elizaarrarás, M.E.; Shapiro, M.; Struppa, D.C.; Vajiac, A. Bicomplex numbers and their elementary functions. Cubo 2012, 14, 61-80.

11. Choi, J.; Datta, S.K.; Biswas, T.; Islam, N. Some fixed point theorems in connection with two weakly compatible mappings in bicomplex valued metric spaces. Honam Math. J. 2017, 39, 115-126. [CrossRef]

12. Jebril, I.H.; Datta, S.K.; Sarkar, R.; Biswas, N. Common fixed point theorems under rational contractions for a pair of mappings in bicomplex valued metric spaces. J. Interdiscip. Math. 2019, 22, 1071-1082. [CrossRef]

13. Dhivya, P.; Marudai, M. Common fixed point theorems for mappings satisfying a contractive condition of rational expression on a ordered complex partial metric space. Cogent Math. 2017, 4, 1389622. [CrossRef]

14. Gunaseelan, M.; Mishra, L.N. Coupled fixed point theorems on complex partial metric space using different type of contractive conditions. Sci. Publ. State Univ. Novi Pazar Ser. A Appl. Math. Inform. Mech. 2019, 12, 117-123. [CrossRef]

15. Gunaseelan, M.; Arul Joseph, G.; Yongji, L.; Zhaohui, G. The existence and uniqueness solution of nonlinear Integral equations via common fixed point theorems. Mathematics 2021, 9, 1179. [CrossRef]

16. Beg, I.; Kumar Datta, S.; Pal, D. Fixed point in bicomplex valued metric spaces. Int. J. Nonlinear Anal. Appl. 2021, 12, 717-727. ISSN 2008-6822. . 